\title{
Performance of a buoyancy-modified $k-\omega$ and $k-\omega$ SST turbulence model for simulating wave breaking under regular waves using OpenFOAM ${ }^{\circledR}$
}

\author{
Brecht Devolder ${ }^{a, b, *}$, Peter Troch ${ }^{a}$, Pieter Rauwoens ${ }^{b}$ \\ a Ghent University, Department of Civil Engineering, Technologiepark 904, 9052 Ghent, Belgium \\ ${ }^{\mathrm{b}} \mathrm{KU}$ Leuven, Department of Civil Engineering, Construction Technology Cluster, Campus Bruges, Spoorwegstraat \\ 12,8200 Bruges, Belgium
}

* Corresponding author. Tel.: +3292645489.

E-mail addresses: Brecht.Devolder@UGent.be (B. Devolder), Peter.Troch@UGent.be (P. Troch), Pieter.Rauwoens@KULeuven.be (P.Rauwoens).

\begin{abstract}
In this work, the performance of a buoyancy-modified turbulence model is shown for simulating wave breaking in a numerical wave flume. Reynolds-Averaged Navier-Stokes (RANS) modelling is performed by applying both a $k-\omega$ and a $k-\omega S S T$ turbulence model using the Computational Fluid Dynamics (CFD) toolbox OpenFOAM. In previous work of the authors (Devolder et al., 2017), the observed significant decrease in wave height over the length of the numerical wave flume based on RANS turbulence modelling for the case of propagating waves has been avoided by developing a buoyancy-modified $k-\omega S S T$ model in which (i) the density is explicitly included in the turbulence transport equations and (ii) a buoyancy term is added to the turbulent kinetic energy (TKE) equation. In this paper, two buoyancy-modified turbulence models are applied for the case of wave breaking simulations: $k-\omega$ and $k-\omega S S T$. Numerical results of wave breaking under regular waves are validated with experimental data measured in a wave flume by Ting and Kirby (1994). The numerical results show a good agreement with the experimental measurements for the surface elevations, undertow profiles of the horizontal velocity and turbulent kinetic energy profiles. Moreover, the underlying motivations for the concept of a buoyancy-modified turbulence model are demonstrated by the numerical results and confirmed by the experimental observations. Firstly, the buoyancy term forces the solution of the flow field near the free water surface to a laminar solution in case of wave propagation. Secondly in the surf zone where waves break, the buoyancy term goes to zero and a fully turbulent solution of the flow field is calculated. Finally and most importantly, the buoyancy-modified turbulence models significantly reduce the common overestimation of TKE in the flow field.
\end{abstract}

Keywords:

CFD; OpenFOAM/IHFOAM; Buoyancy-modified turbulence model; Wave breaking

Postprint accepted for publication in Coastal Engineering, August 2018, vol. 138, pp. 49-65.

https://doi.org/10.1016/j.coastaleng.2018.04.011 


\section{Introduction}

Wave breaking is seen as one of the last and most complex life events of a wave. When waves propagate from offshore towards the shoreline, shoaling occurs due to a decreasing water depth. In the surf zone, the wave steepness increases significantly and the waves break. Wave breaking is characterised by several parameters such as the wave height $H$, the wave length $L$ and the bathymetry of the seabed. Therefore, Galvin [1] reported four different breaker types: spilling, plunging, surging and collapsing. During wave breaking, turbulence generation is one of the governing processes. However, it is very challenging to quantify wave breaking turbulence based on field observations. Therefore, a large number of experimental flume tests have been performed in a repeatable and controlled environment studying both regular and irregular wave breaking, e.g. [2-13]. Those flume tests revealed that wave breaking is the primary source of turbulence generation and is responsible for the dissipation of wave energy. In particular, wave breaking events increase the turbulent intensity in the vicinity of the seabed, which plays an important role in near shore sediment transport. Next to experimental modelling, numerical simulations provide additional insights since a lot of difficulties for measuring wave breaking characteristics exist during field measurements or small-scale experimental tests. In particular, the disturbance effect of measurement devices on the hydrodynamic flow field and the influence of entrapped air on measuring surface elevations and velocity profiles are the main contributions to the overall error in the experimentally obtained results.

The focus of the present numerical study is solely put on wave breaking induced turbulence modelling using a Navier-Stokes solver and not on sediment transport. Numerical studies regarding sediment transport under breaking wave conditions have been reported in [14-17] for example. Furthermore, long duration tests using irregular waves are not considered in this numerical validation study in order to minimise the computational effort. In this paper, Ting and Kirby [2] is selected as the experimental validation dataset since a rigid seabed was used and regular waves were generated. The experimental dataset contains measurements of both surface elevations and undertow profiles for spilling and plunging breakers respectively. Several authors reported numerical results using that experimental dataset, such as Lin and Liu [18], Bradford [19], Mayer and Madsen [20], Christensen [21], Hieu et al. [22], Jacobsen et al. [23], Xie [24], Alagan Chella et al. [25,26] and Brown et al. [27]. All the numerical studies have one aspect in common: they all use a turbulence model in their Navier-Stokes solver. The need for using a turbulence model is motivated by e.g. Thornton [28]: wave breaking is a paramount source of turbulence generation. Over the past decades, a large number of turbulence models have been derived and modified in order to enhance the predictive skills of numerical methods for turbulent fluid flows. Simulating surf zone turbulence specifically requires attention to the choice of a turbulence model. For the first numerical studies simulating wave breaking, Reynolds-Averaged Navier-Stokes (RANS) models and in particular $k-\varepsilon$ models were popular [18,19]. [18] developed a RANS model using an algebraic Reynolds stress $k-\varepsilon$ model in combination with a Volume of Fluid (VoF) method to capture the free water surface. Their model was able to simulate the turbulence levels and the mean flow field in the surf zone away from the breaking point very well, even though their simulation did not reach the quasi-steady state. However, the authors found that the turbulence levels near the breaking point were significantly overestimated compared to the experimental data. As a consequence, energy dissipation was responsible for the smaller observed breaking wave height. [19] performed RANS simulations using the commercial software Flow-3D by applying different one- and two-equation turbulence models: $k$-model, $k-\varepsilon$ model, RNG model. The results obtained using the one-equation $k$-model were insufficient accurate, while the performance of the RNG model was lower than the $k-\varepsilon$ model for predicting turbulence levels. In general, [19] concluded that the turbulent kinetic energy (TKE) in the wave crest prior to breaking is overpredicted using a $k-\varepsilon$ model, resulting in an underprediction of the breaking wave height. Therefore, [19] advises to use a $k-\omega$ model instead, as 
presented by [20]. However, [20] modified the $k-\omega$ model in order to overcome the generation of TKE in the potential flow region (i.e. wave propagation zone outside the surf zone) and to avoid wave damping over the length of the flume. Therefore, they implemented an ad-hoc modification of the production of TKE using the vorticity of the mean flow rather than the local mean velocity gradient. As a result, this modification eliminated the excessive generation of TKE outside the surf zone when using a $k-\omega$ model and enhanced significantly the predictive skills of the numerical model for simulating breaking waves. Their research also triggered the discussion on the fundamental problems of applying RANS models for wave modelling. An alternative approach to RANS models are large eddy simulations (LES). In LES, the large scale turbulent structures are computed directly while the small scale (sub-grid scale) structures are modelled using a turbulence model. [29] formulated that at least $80 \%$ of the turbulence needs to be resolved by the computational grid for LES. As a consequence, very fine grids are needed, increasing the computational time significantly. [22] presented two-dimensional (2D) LES calculations using a two-phase flow solver for simulating breaking waves and satisfactory results were obtained for a limited simulation time. In their model, not only the water phase but also the air phase was modelled. They noted that the effects of air entrainment on the wave energy dissipation were not negligible. Furthermore, they reported that surface tension might be necessary for better simulations of air bubbles entrained in the water. Thereafter, [21] presented three-dimensional (3D) LES calculations for modelling both spilling and plunging breakers. In his model the air phase was not considered at all in order to take the air-water mixture into account in the surf zone. In general, a fair agreement was found between numerical and experimental surface elevations in the inner part of the surf zone. However, the exact breaking point was not captured accurately and the breaking wave height was overestimated by the LES model. Also the undertow profiles showed discrepancies between experimental and numerical results. [21] concluded that the differences between numerical and experimental results were mainly due to the coarse resolution of the 3D mesh. Note that not only [21] but also [18-20] used a single-phase fluid solver. However, single-phase solvers are not able to reproduce the energy dissipation caused by entrained air, as reported in $[14,21,22]$, resulting in an overprediction of TKE ([18-21]). More recently, [23] presented a wave generation toolbox for the twophase flow solver implemented in OpenFOAM and performed validation tests for breaking waves using the modified $k$ - $\omega$ model proposed by [20]. The results presented are averaged over 50 wave periods after the warming-up phase of 80 wave periods in order to avoid the apparent lack of mass conservation. Furthermore, [23] demonstrated the importance of the aspect ratio on the numerical results. In general, the numerical results for the surface elevations and undertow profiles obtained with an aspect ratio equal to 1 were significantly better compared to an aspect ratio equal to 2. [24] used a two-phase flow solver with a $k-\varepsilon$ model but only a limited number of waves were simulated. In general, their results showed a good agreement with the experimental data. The numerically obtained breaking wave height was under estimated by the numerical model while good results were obtained for the TKE levels. As an alternative to the VoF method, $[25,26]$ applied a Level Set Method (LSM) to track the free water surface in combination with a $k-\omega$ model implemented in the two-phase flow solver REEF3D. At the interface between water and air, a turbulence damping scheme was applied to avoid unphysical turbulence production. Their model predicted shoaling wave heights very well, however discrepancies were observed for the surface elevations in the surf zone. In contrast to all other numerical studies, their model underpredicted the TKE levels in the surf zone. Presumably, this was caused by applying a turbulence damping scheme everywhere near the free water surface, not only in the wave propagation zone but also in the surf zone. Consequently, the wave breaking induced turbulence generation was severely restricted. Very recently, [27] reported an overview of using various turbulence models in OpenFOAM to simulate both spilling and plunging breakers. Moreover, the authors addressed the necessity of including the density explicitly in the turbulence transport equations. After implementing the density explicitly in the turbulence transport equations, [27] 
concluded that the overall best model is the nonlinear $k-\varepsilon$ model but the $k$ - $\omega$ model showed improvements for all the results compared to a solution without turbulence model.

Based on a review of those previous studies, two knowledge gaps are defined. Firstly, it is clear that no standard turbulence model exists for simulating breaking waves. In this study, we propose to test a widely-known Reynolds-Averaged Navier-Stokes (RANS) turbulence model to evaluate its performance during wave breaking simulations using a two-phase Navier-Stokes solver. Regarding the $k-\varepsilon$ model, it is known that the transport equation for $\varepsilon$ becomes singular near the wall when it is integrated through the viscous sublayer. This singularity is treated with damping functions but they feature stability issues [30]. A robust alternative formulation is the $k-\omega$ model of Wilcox [31]. It has the advantage of an accurate near wall treatment without employing damping functions. The numerical stability is improved due to straightforward Dirichlet boundary conditions near the wall. Consequently, those two advantages have a direct impact on choosing a $k-\omega$ model to simulate breaking waves on a sloping beach because the bottom will have an influence on the wave breaking process. However, it is reported by Menter [32] that the results obtained with a $k-\omega$ model strongly depend on the freestream values of $\omega$ outside the boundary layer in case of free shear layers. Therefore, Menter [30] developed a $k-\omega$ shear stress transport (SST) model in order to overcome the freestream dependency of $\omega$. In that model, blending functions are applied in order to activate the $k-\omega$ model in the inner region of the boundary layer and the $k-\varepsilon$ model in the outer and free shear region. In this paper, both the $k-\omega$ and $k-\omega S S T$ models are applied for the numerical simulations presented. Secondly, the review of previous studies emphasises the need for enhanced prediction tools to simulate accurately the turbulence levels in the flow field of surf zone and in particular near the breaking point. Many researchers, such as $[14,16,17,21,24,27]$, reported that the TKE in flow field is numerically overpredicted, except for [26] who observed underpredictions. Moreover, it is generally known that none of the traditional turbulence models (e.g. $k-\varepsilon, k-\omega$ and $k-\omega S S T$ ) are developed for two-phase flow simulations (such as wave breaking) but for an incompressible single phase flow. For example, the $k$ - $\omega$ model is originally developed for aerodynamic and aerospace applications. Therefore, these traditional models have to be modified to account for the effect of density variations in the numerical wave flume. In general for a two-phase flow solver, the density should be included in the turbulence transport equations. For the $k-\omega$ model in particular, [23] also applied a revised production term for the TKE in order to enhance its prediction in the flow field. However, [20] did not advise this modification as generally valid and recommended fundamental analysis and developments.

In general for wave breaking simulations in a numerical wave flume, two zones are distinguished: the wave propagation zone (no turbulence model needed) and the surf zone (turbulence model needed). Consequently, the numerical wave flume needs to be split up and separate (coupled) simulations have to be performed. However in this paper, we propose a unified model that can handle both regions at the same time. Therefore, we not only include the density in the turbulence transport equations but we also account for the effect caused by density variations in a two-phase flow. The latter is realised by adding a buoyancy source term in the TKE-equation as introduced in [33]. Note that [18-21] all used a single-phase fluid solver and consequently, they did not need a buoyancy correction in the TKEequation to account for density variations in the numerical wave flume. However, as stated before, single-phase fluid solvers overpredict TKE. For a two-phase fluid solver, the buoyancy term is not an ad-hoc modification but appears when the transport equation for the TKE is fundamentally derived from the Favre-averaged (density weighted) low Mach number equations, as reported in $[34,35]$. The similarity in terms of a varying density between our research and the fire flows studied in $[34,35]$ was already clarified in our previous work [33]. Furthermore as reported in [33], the RANS approach using a two-phase flow solver might cause a significant decrease in wave height over the length of the numerical wave flume for wave propagation simulations. Moreover, we pointed out the need for a 
buoyancy-modified turbulence model to simulate both low and high steepness propagating waves in non-breaking conditions. Therefore, a buoyancy term was implemented in the turbulent kinetic energy (TKE) equation of the $k-\omega S S T$ model inducing a laminar result near the free water surface. This new implementation resulted in an overall stable wave propagation model without a significant decrease in wave height over the length of the flume.

In order to fill the knowledge gaps, the focus of this paper is put on the performance of our buoyancymodified $k-\omega$ and $k-\omega$ SST models for wave breaking processes. The first question which will be addressed for the case of wave breaking simulations is whether the zone specifically requiring a turbulence model (i.e. the surf zone) is still well predicted with a buoyancy-modified turbulence model? In other words: is a fully turbulent flow field resolved at the locations where wave breaking is happening? Secondly by using a buoyancy-modified turbulence model, can we solve the issue of overpredicting the TKE in the two-phase flow field? Therefore in this paper, we present a study on the performance of buoyancy-modified turbulence models for simulating wave breaking under regular waves using the Computational Fluid Dynamics (CFD) toolbox OpenFOAM ${ }^{\circledR}$ [36]. Numerical simulations are performed and compared with the experimental dataset of Ting and Kirby [2] for both spilling and plunging breakers on a plane slope (1:35). RANS turbulence modelling is performed by applying both the original and buoyancy-modified $k-\omega$ and $k-\omega$ SST models.

The remainder of this paper is organised as follows. Firstly, in section 2, the governing equations for the numerical model are presented, followed by a description of the computational domain, the boundary conditions applied and the solver settings. Subsequently in section 3 , the numerical model is used to perform wave breaking simulations and the numerical results are compared to experimental measurements while in section 4 the obtained numerical results are discussed in detail. Finally, the conclusions are drawn in section 5.

\section{Numerical model}

The numerical simulations presented are achieved using OpenFOAM ${ }^{\circledR}$ [36], version 2.2.2. Firstly, the flow equations are introduced, followed by a description of turbulence modelling. Subsequently, the computational domain is presented together with the grid characteristics. The last two parts of this section are dedicated to explain the different boundary conditions and solver settings.

\subsection{Flow equations}

The numerical model uses the incompressible RANS equations to express the motion of a fluid consisting of a mass conservation equation (1) and a momentum conservation equation (2) written in Einstein summation notation as:

$$
\begin{gathered}
\frac{\partial u_{i}}{\partial x_{i}}=0 \\
\frac{\partial \rho u_{i}}{\partial t}+\frac{\partial \rho u_{j} u_{i}}{\partial x_{j}}-\frac{\partial}{\partial x_{j}}\left[\mu_{e f f} \frac{\partial u_{i}}{\partial x_{j}}\right]=-\frac{\partial p^{*}}{\partial x_{i}}+F_{b, i}+f_{\sigma, i}
\end{gathered}
$$

in which $t$ is the time, $u_{i}(i=x, y, z)$ are the Cartesian components of the fluid velocity, $\rho$ is the fluid density, $\mu_{\text {eff }}$ is the effective dynamic viscosity, $p^{*}$ is the pressure in excess of the hydrostatic. $F_{b}$ is an external body force (including gravity) and $f_{\sigma}$ is the surface tension tensor term which are respectively defined as:

$$
\begin{gathered}
F_{b, i}=-g_{i} x_{i} \frac{\partial \rho}{\partial x_{i}} \\
f_{\sigma, i}=\sigma \kappa \frac{\partial \alpha}{\partial x_{i}}
\end{gathered}
$$


in which vector $\vec{g}=[0 ; 0 ;-9.81] \mathrm{m} / \mathrm{s}^{2}, \vec{x}$ is the Cartesian coordinate vector $(x, y, z), \sigma$ is the surface tension coefficient, $k$ is the mean curvature of the interface and $\alpha$ is the volume fraction. Note that the mean values for the variables considered are written in terms of Favre-averaging (density weighted) due to the varying density.

The interface between water and air is obtained by the VoF method as documented in [37]. This enhanced formulation using a compression term reduces the dissipative nature of the interface compared to the VoF method of [38]. The method is based on a volume fraction $\alpha$ which is 0 for a completely dry cell and 1 for a completely wet cell and in between 0 and 1 for an interface cell containing both water and air. The volume fraction is solved by an advection equation (5):

$$
\frac{\partial \alpha}{\partial t}+\frac{\partial u_{i} \alpha}{\partial x_{i}}+\frac{\partial u_{c, i} \alpha(1-\alpha)}{\partial x_{i}}=0
$$

The last term on the left-hand side is an artificial compression term where $u_{c, i}=\min \left[c_{\alpha}\left|u_{i}\right|, \max \left(\left|u_{i}\right|\right)\right]$. In the present study, the default value of $c_{\alpha}$ equal to 1 is applied. If a larger value is used, the compression of the interface increases, leading to larger detrimental velocity gradients around that interface.

The density of the fluid $\rho$ within a computational cell is calculated by a weighted value based on the volume fraction $\alpha$. The effective dynamic viscosity $\mu_{\text {eff }}$ is obtained by the sum of a weighted value based on the volume faction $\alpha$ and an additional turbulent dynamic viscosity $\rho v_{t}$ :

$$
\begin{gathered}
\rho=\alpha \rho_{\text {water }}+(1-\alpha) \rho_{\text {air }} \\
\mu_{\text {eff }}=\alpha \mu_{\text {water }}+(1-\alpha) \mu_{\text {air }}+\rho v_{t}
\end{gathered}
$$

In a post processing step, the position of the free water surface is determined by a discrete integration of the volume fraction $\alpha$ over a vertical line (Z-direction) divided in $n$ equal parts:

$$
z_{\text {water level }}=\sum_{i=0}^{n-1} \alpha_{i}\left(z_{i+1}-z_{i}\right)
$$

\subsection{Turbulence modelling}

Turbulent effects are incorporated in the RANS equations (1) and (2) by solving one or more additional transport equations to yield a value for the turbulent kinematic viscosity $v_{t}$. Once the turbulent viscosity is known, the Reynolds stress tensor can be calculated in OpenFOAM ${ }^{\circledR}$ [36] as:

$$
\tau_{i j}=\frac{2}{3} k \delta_{i j}-v_{t}\left(\frac{\partial u_{i}}{\partial x_{j}}+\frac{\partial u_{j}}{\partial x_{i}}\right)
$$

where $k$ is the turbulent kinetic energy, $\delta_{i j}$ is the Kronecker delta and $v_{t}$ is the turbulent kinematic viscosity.

In the present study, a $k-\omega$ and a $k-\omega S S T$ model are tested regarding their performance for wave breaking simulations. The results obtained with the original implemented versions of both turbulence models in OpenFOAM ${ }^{\circledR}$ [36] and their buoyancy-modified versions are compared with the experimental dataset.

\subsubsection{Incompressible $k-\omega$ model}

The incompressible $k-\omega$ model for a single fluid is a two-equation model [31] and is formulated in OpenFOAM $^{\circledR}[36]$ as:

$$
\frac{\partial k}{\partial t}+\frac{\partial u_{j} k}{\partial x_{j}}-\frac{\partial}{\partial x_{j}}\left[\left(v+\sigma_{k} v_{t}\right) \frac{\partial k}{\partial x_{j}}\right]=P_{k}-\beta^{*} \omega k
$$




$$
\begin{gathered}
\frac{\partial \omega}{\partial t}+\frac{\partial u_{j} \omega}{\partial x_{j}}-\frac{\partial}{\partial x_{j}}\left[\left(v+\sigma_{\omega} v_{t}\right) \frac{\partial \omega}{\partial x_{j}}\right]=\gamma \frac{\omega}{k} P_{k}-\beta \omega^{2} \\
P_{k}=v_{t} \frac{\partial u_{i}}{\partial x_{j}}\left(\frac{\partial u_{i}}{\partial x_{j}}+\frac{\partial u_{j}}{\partial x_{i}}\right) \quad v_{t}=\frac{k}{\omega}
\end{gathered}
$$

where $k$ is the turbulent kinetic energy, $P_{k}$ is the production term of $k, v$ is the kinematic viscosity, $v_{t}$ is the turbulent kinematic viscosity, $\omega$ is the specific dissipation rate, $\sigma_{k}=\sigma_{\omega}=0.5, \beta^{*}=0.09, \beta=0.072$ and $y=0.52$.

\subsubsection{Incompressible $k$ - $\omega$ SST model}

The incompressible $k$ - $\omega S S T$ model for a single fluid is a two-equation model [39] and is formulated in OpenFOAM ${ }^{\circledR}[36]$ as:

$$
\begin{gathered}
\frac{\partial k}{\partial t}+\frac{\partial u_{j} k}{\partial x_{j}}-\frac{\partial}{\partial x_{j}}\left[\left(v+\sigma_{k} v_{t}\right) \frac{\partial k}{\partial x_{j}}\right]=P_{k}-\beta^{*} \omega k \\
\frac{\partial \omega}{\partial t}+\frac{\partial u_{j} \omega}{\partial x_{j}}-\frac{\partial}{\partial x_{j}}\left[\left(v+\sigma_{\omega} v_{t}\right) \frac{\partial \omega}{\partial x_{j}}\right]=\frac{\gamma}{v_{t}} G-\beta \omega^{2}+2\left(1-F_{1}\right) \frac{\sigma_{\omega 2}}{\omega} \frac{\partial k}{\partial x_{j}} \frac{\partial \omega}{\partial x_{j}} \\
P_{k}=\min \left(G, 10 \beta^{*} k \omega\right) \quad G=v_{t} \frac{\partial u_{i}}{\partial x_{j}}\left(\frac{\partial u_{i}}{\partial x_{j}}+\frac{\partial u_{j}}{\partial x_{i}}\right) \quad v_{t}=\frac{a_{1} k}{\max \left(a_{1} \omega, S F_{2}\right)}
\end{gathered}
$$

where $k$ is the turbulent kinetic energy, $P_{k}$ is the production term of $k, v$ is the kinematic viscosity, $v_{t}$ is the turbulent kinematic viscosity, $\omega$ is the specific dissipation rate, $S$ is the mean rate of strain of the flow, $\beta^{*}=0.09$ and $a_{1}=0.31$. $F_{1}$ and $F_{2}$ are blending functions. $F_{1}$ is designed to be one in the near wall region (activating $k-\omega$ ) and zero away from the wall (activating $k-\varepsilon$ ). The values of $\sigma_{k}, \sigma_{\omega}, \beta$ and $\gamma$ are blended using equation (16) in which $\phi_{1}$ and $\phi_{2}$ are given in Table 1.

$$
\phi=F_{1} \phi_{1}+\left(1-F_{1}\right) \phi_{2}
$$

Table 1: Default values for $\phi_{1}$ and $\phi_{2}$ used in equation (16) to calculate $\sigma_{k}, \sigma_{\omega}, \beta$ and $y$ for the $k-\omega$ SST model.

\begin{tabular}{c|cccc}
\hline$\phi$ & $\sigma_{k}$ & $\sigma_{\omega}$ & $\beta$ & $\gamma$ \\
\hline$\phi_{1}$ & 0.85034 & 0.5 & 0.075 & 0.5532 \\
\hline$\phi_{2}$ & 1.0 & 0.85616 & 0.0828 & 0.4403 \\
\hline
\end{tabular}

\subsubsection{Buoyancy-modified $k-\omega$ and $k-\omega S S T$ models}

As reported in [33], the original $k$ - $\omega$ SST model causes significant wave damping for non-breaking propagating high steepness waves. This damping is triggered by an increase in turbulent viscosity around the interface between water and air. This increase is induced by the large production of turbulent kinetic energy (TKE), $k$, in that zone. The production of TKE is linked to the velocity gradient which is large around the interface between water and air due to spurious air velocities. Those spurious air velocities arise due to the pressure-density coupling resolved in the conditionally averaged momentum equation using segregated solution algorithms [40]. Consequently, a natural imbalance exists between the pressure gradient and the large density gradient at the free water surface due to the large density ratio (1000/1). A recent paper addressing the spurious velocities is e.g. [40]. In this study however, both a buoyancy-modified $k-\omega$ and $k-\omega S S T$ model are implemented in OpenFOAM by:

i. including the density $\rho$ explicitly in the equations (10) (11) (13) (14);

ii. adding a buoyancy term $G_{b}$ in the TKE-equations (10) and (13). The term is not included in the equation for $\omega$ since not much influence is expected on the results [33].

The buoyancy-modified $k$ - $\omega$ model is defined as: 


$$
\begin{aligned}
& \frac{\partial \rho k}{\partial t}+\frac{\partial \rho u_{j} k}{\partial x_{j}}-\frac{\partial}{\partial x_{j}}\left[\rho\left(v+\sigma_{k} v_{t}\right) \frac{\partial k}{\partial x_{j}}\right]=\rho P_{k}+G_{b}-\rho \beta^{*} \omega k \\
& \frac{\partial \rho \omega}{\partial t}+\frac{\partial \rho u_{j} \omega}{\partial x_{j}}-\frac{\partial}{\partial x_{j}}\left[\rho\left(v+\sigma_{\omega} v_{t}\right) \frac{\partial \omega}{\partial x_{j}}\right]=\gamma \frac{\omega}{k} \rho P_{k}-\rho \beta \omega^{2}
\end{aligned}
$$

The buoyancy-modified $k-\omega S S T$ model is defined as:

$$
\begin{array}{r}
\frac{\partial \rho k}{\partial t}+\frac{\partial \rho u_{j} k}{\partial x_{j}}-\frac{\partial}{\partial x_{j}}\left[\rho\left(v+\sigma_{k} v_{t}\right) \frac{\partial k}{\partial x_{j}}\right]=\rho P_{k}+G_{b}-\rho \beta^{*} \omega k \\
\frac{\partial \rho \omega}{\partial t}+\frac{\partial \rho u_{j} \omega}{\partial x_{j}}-\frac{\partial}{\partial x_{j}}\left[\rho\left(v+\sigma_{\omega} v_{t}\right) \frac{\partial \omega}{\partial x_{j}}\right] \\
=\frac{\gamma}{v_{t}} \rho G-\rho \beta \omega^{2}+2\left(1-F_{1}\right) \rho \frac{\sigma_{\omega 2}}{\omega} \frac{\partial k}{\partial x_{j}} \frac{\partial \omega}{\partial x_{j}}
\end{array}
$$

The buoyancy term $G_{b}$ is defined as:

$$
G_{b}=-\frac{v_{t}}{\sigma_{t}} \frac{\partial \rho}{\partial x_{j}} g_{j}
$$

in which the scalar $\sigma_{t}=0.85$ [33]. The buoyancy term $G_{b}$ is treated implicitly in the TKE-equation because $v_{t}$ is equal to $k / \omega$, see equations (12) and (15).

The purpose of including a buoyancy term in this study is twofold. The first objective is to suppress the turbulence level at the free water surface, i.e. in the zone where the governing direction of the density gradient is vertical (predominantly horizontal free water surface). More specific, this is the zone near the interface where non-breaking waves are propagating. Because of the implicit treatment of the buoyancy term $G_{b}$ in the TKE-equation, the very large vertical density gradient near the free water surface drives the turbulent viscosity $v_{t}$ to zero. As a result, in case of propagating waves, the model switches to a laminar regime near the free water surface, preventing excessive wave damping. This has already been demonstrated in [33]. The second objective is to obtain a fully turbulent solution of the flow field in the surf zone, i.e. in the zone where the density gradient consists of an important horizontal component. At the breaking point, this condition is obtained when shoaling waves are reaching their limiting wave height. In the limit of a vertical wave front, $G_{b}$ is equal to 0 and consequently the original turbulence model formulation (including the density but without buoyancy modification) is regained.

\subsection{Computational domain}

For this study a two-dimensional simulation in a vertical plane (2DV) is performed. Although wave breaking is a three-dimensional process, a 2DV model is able to simulate the governing wave breaking characteristics with a reasonable accuracy as shown by several other 2DV numerical studies in literature [18-20,22-27]. In particular, [17] reported a comparison between 2DV and 3D results. They concluded that wave breaking starts form $2 \mathrm{D}$ horizontal rollers and subsequently evolves into full $3 \mathrm{D}$ hairpin-shaded turbulent structures causing cross-sectional variability in the flow field. As a result, discrepancies between 2DV and 3D models are mainly observed in the inner surf zone [17]. Figure 1 shows the computational domain together with the boundary condition types which are listed in the next section 2.4. The waves are generated at the inlet in a water depth of $0.40 \mathrm{~m}$ and propagate first over a horizontal bed (1.3 m long) and subsequently over a uniform slope of 1:35 in order to replicate the experimental tests. 
After uniform discretisation, the largest size of a cell in both the horizontal $X$-direction $(\Delta x)$ and vertical $Z$-direction $(\Delta z)$ is $0.01 \mathrm{~m}$. Subsequently, the mesh is locally refined in horizontal and vertical direction in a zone where the free water surface will be located, resulting in $\Delta x=\Delta z=0.005 \mathrm{~m}$. In general, the aspect ratio (i.e. ratio of largest dimension of a cell over the smallest dimension) is 1 as suggested by [23]. However, additional layers with $\Delta x=0.005 \mathrm{~m}$ are added next to the bottom boundary in order to resolve the boundary layer properly, locally increasing the aspect ratio up to 8 . These cell sizes are sufficiently small based on the mesh refinement study of [27]. The final grid consists of 329946 cells and 360594 cells for the spilling and plunging breakers respectively.

\subsection{Boundary conditions}

The types of boundary conditions for this 2DV simulation are given in Figure 1: bottom, atmosphere on the top, inlet on the left and outlet on the right. The bottom is modelled as a smooth solid wall on which wall functions are activated for $k$ and $\omega$. A continuous wall function based on Spalding's law [41] switching between low- and high-Reynolds numbers is implemented for the turbulent viscosity $v_{t}$. By using this scalable wall function, the dimensionless wall distance $y^{+}$should be between 1 and 300 . The initial values for $k$ and $\omega$ in the computational domain are set to $10^{-6} \mathrm{~m}^{2} / \mathrm{s}^{2}$ and $1.0 \mathrm{~s}^{-1}$ respectively. Furthermore on the bottom, a Dirichlet boundary condition is set for the velocity $(0 \mathrm{~m} / \mathrm{s}$ in the two directions) while the pressure and volume fraction are set to a Neumann condition. The atmospheric conditions at the top of the numerical domain are set to a mixed Dirichlet-Neumann boundary condition for the velocity, pressure and volume fraction. At the inlet, a special boundary condition is needed to generate the incoming and absorb the reflected waves. IHFOAM $[42,43]$ is deployed as an external toolbox for that boundary condition. Both wave generation and active wave absorption are activated at the inlet. Wave reflection is not observed at the outlet because no water will reach that boundary. Therefore, a fixed wall boundary condition is implemented at the outlet boundary, similar to the bottom boundary.

In case of spilling breakers, a wave height $H=0.125 \mathrm{~m}$ is generated with a wave period $T=2 \mathrm{~s}$ resulting in a breaker parameter $\xi_{0}=m /\left(H_{0} / L_{0}\right)^{1 / 2}=0.20$ based on deep water conditions for $H$ and $L$, and $m$ is the bottom slope (1:35) [2]. In case of plunging breakers, the wave height $H=0.127 \mathrm{~m}$ and the wave period $T=5 \mathrm{~s}$ resulting in a breaker parameter $\xi_{0}=m /\left(H_{0} / L_{0}\right)^{1 / 2}=0.60$ [2]. For both cases, the water depth $d$ at the wave generating boundary is fixed to $0.40 \mathrm{~m}$. Stream function theory is used for the generation of both spilling and plunging breakers in the numerical wave flume, and the Stokes velocity is set to zero (i.e. no mass transported in a closed wave flume).

\subsection{Solver settings}

For all the simulations presented, the following solver settings are used: central discretisation for the pressure gradient and the diffusion terms; TVD (total variation diminishing) schemes with a van Leer limiter [44] for the divergence operators; backward Euler time discretisation; a maximum Courant number equal to 0.20 (spilling breakers) and 0.10 (plunging breakers) [27].

\section{Results}

In the following two subsections, the numerically obtained surface elevations, undertow profiles and TKE profiles are presented and compared to experimental measurements from Ting and Kirby [2] for both spilling and plunging breakers respectively. Each numerical simulation ran for 50 wave periods to obtain a sufficiently long dataset after the warming-up phase. All the results presented in this section are phase averaged using the last 20 waves of the 50 waves simulated.

Five simulations are performed using no turbulence model, the original $k-\omega$ and $k-\omega$ SST models and the buoyancy-modified $k-\omega$ and $k-\omega$ SST models. Additionally, numerical results of the surface 
elevations using the $k-\omega$ and $k-\omega S S T$ models in which only the density is included are also provided. Those results are obtained by switching off the buoyancy term: $G_{b}=0$ in equations (17) and (19). The result without turbulence model is included to address the need of turbulence modelling for the case of breaking waves. The maximum achieved $y^{+}$values on the bottom of the numerical wave flume are summarised in Table 2 for both spilling and plunging breakers using the six different turbulence models. Those $y^{+}$values are within the application range reported in section 2.4 .

Table 2: $y^{+}$values on the bottom of the numerical wave flume for both spilling and plunging breakers using the six different turbulence models.

\begin{tabular}{c|cc|c|c|cc}
\hline \multirow{2}{*}{$y^{+}$} & \multicolumn{2}{|c|}{ original } & \multicolumn{2}{c|}{ density only } & \multicolumn{2}{c}{ buoyancy-modified } \\
\cline { 2 - 7 } & $k-\omega$ & $k-\omega S S T$ & $k-\omega$ & $k-\omega S S T$ & $k-\omega$ & $k-\omega S S T$ \\
\hline spilling & 36 & 41 & 34 & 31 & 34 & 41 \\
\hline plunging & 30 & 45 & 61 & 41 & 48 & 36 \\
\hline
\end{tabular}

\subsection{Spilling breakers}

In this subsection, both the experimental [2] and numerical results are reported for the case of spilling breakers. Firstly, surface elevations are presented followed by velocities and TKE along several vertical profiles. Lastly, the turbulent behaviour of the flow using different RANS turbulence models is shown.

\subsubsection{Surface elevations}

The graphs displayed in Figure 2 include the surface elevations along the wave flume for the seven simulations. In each graph, the vertical axis denotes the phase averaged surface elevation $\eta$ with respect to the bottom before the slope $(z=0 \mathrm{~m})$. On the horizontal axis, $x=-2 \mathrm{~m}$ corresponds to the inlet boundary while $x=-0.7 \mathrm{~m}$ indicates the start of the slope. The solid blue lines represent the numerical results for the maximum, average and minimum phase averaged surface elevations. The blue shaded bands indicate one standard deviation on both sides of the maximum and minimum surface elevations which represent about $68 \%$ of all the values in case of a normal distribution. The root mean square error (RMSE), denoted by $E$, is calculated with respect to the experimental data (indicated by red dots in Figure 2$)$ for the maximum $\left(E_{\max }\right)$, average $\left(E_{a v}\right)$ and minimum $\left(E_{\min }\right)$ surface elevations respectively. Note that RMSE values are not dimensionless and therefore they are only used to compare in a qualitative way the performance of the various turbulence models. The vertical dashed black lines indicate the position where the vertical profiles are extracted, see section 3.1.2.

In general, Figure 2 addresses the need of using a turbulence model for simulating wave breaking processes. It is clearly observed that the solution without turbulence model gives significantly deviating results for the surface elevations compared to the experimental data. In particular, the numerically obtained breaking point is far away from the experimental one, as is the case for both the average and minimum surface elevations. In general, the results where a turbulence model is used, except for the original $k-\omega$ model and the $k-\omega$ model with only the density included, are in a good agreement with the experimental data both for the location of the breaking point and the surface elevations. This is shown by the smaller RMSE values for the maximum, average and minimum surface elevations using the buoyancy-modified $k-\omega$ model and all the $k-\omega$ SST models, compared to RMSE values for the solution without turbulence model, the original $k-\omega$ model and the $k-\omega$ model with only the density included. In contrast to the solution without applying a turbulence model, turbulence modelling enhances the capability of the numerical model to obtain repeatable waves over consecutive wave periods characterised with low standard deviations (see blue shaded bands in Figure 2). Remarkably, discrepancies between numerical and experimental results are only observed for the maximum surface elevations and not for the average and minimum ones. 
Furthermore, a major difference in the performance between the original $k-\omega$ and $k-\omega S S T$ model is observed. It is clearly shown in Figure 2 that significant wave damping is observed over the length of the flume in case of the original $k-\omega$ model while this is not simulated by the original $k-\omega S S T$ model. This is explained by the limiter inside the production term of TKE: $P_{k}$, see equation (15). The motivation to use a limiter was to avoid excessive generation of turbulent viscosity in the vicinity of stagnation points around an airfoil [30]. As a result in the present study, the production of TKE is limited and hence reducing the turbulent viscosity, avoiding wave damping. Although the original $k-\omega S S T$ model slightly underpredicts the maximum surface elevation at the breaking point, the surface elevations after wave breaking are in a good agreement with the experimental data. If the density is included explicitly in the $k-\omega$ model, wave damping is still observed but not as strong as the original $k-\omega$ model. Surprisingly, [27] did not observe wave damping for the spilling breakers using a $k$ - $\omega$ model in which only the density is included. As expected, a major improvement in surface elevations is observed for the buoyancymodified $k-\omega$ model. Now, the surface elevations are very similar to the experimental data and they all have small standard deviations. Regarding the $k-\omega S S T$ model including only the density and the buoyancy-modified $k-\omega S S T$ model, the maximum surface elevations are slightly better predicted at the breaking point compared to the original $k-\omega S S T$ model. However, smaller and less smooth maximum surface elevations are observed after the waves broke. The small differences in the maximum surface elevations obtained by the three $k-\omega S S T$ models are also explained by the limiter inside the production term of TKE. As a result, the buoyancy-modified $k-\omega$ model has the best performance in terms of surface elevations for the case of spilling breakers. Moreover, it is also shown that the inclusion of the buoyancy term is essential for a $k-\omega$ model in order to avoid wave damping.

\subsubsection{Undertow profiles}

Figure 3 presents both measured and simulated undertow profiles at locations $x=-1.265 \mathrm{~m}, x=$ $5.945 \mathrm{~m}, x=6.665 \mathrm{~m}, x=7.275 \mathrm{~m}, x=7.885 \mathrm{~m}, x=8.495 \mathrm{~m}, x=9.110 \mathrm{~m}$ and $x=9.725 \mathrm{~m}$ (see vertical dashed lines in Figure 2). Along those vertical profiles, the calculated time averaged horizontal velocity $\bar{u}$ and time averaged turbulent kinetic energy (TKE) $\bar{k}$ are analysed. Note that in this work, only the mean value for TKE, $\bar{k}$, is studied. [45] presented an analysis of the mean turbulence from the resolved flow field to the total TKE and observed that the modelled TKE by a RANS turbulence model was of larger importance. A similar analysis was performed in this study and a similar conclusion is obtained. Consequently, only the mean value for TKE, $\bar{k}$, modelled by a RANS turbulence model is presented in the remainder of this study. Numerical results are shown in solid blue lines whereas the discrete experimental data are indicated by red dots. On top of each curve, the RMSE $E$ is reported with respect to the experimental results. Numerical results using no turbulence model and the original $k$ - $\omega$ model are excluded because the surface elevations shown in Figure $2 a$ and Figure $2 b$ are inaccurate.

In general, all three simulations presented in Figure 3 predict a good qualitative behaviour of the undertow: i.e. negative values for $\bar{u}$ over the largest part of the water column and $\bar{u}$ becomes positive near the free water surface. Furthermore, the transition from positive to negative is captured correctly for all the profiles compared to the experimental data. However, near the bottom, deviations become visible among the turbulence models for the profiles located at $x \geq 7.275 \mathrm{~m}$. A comparison of the original $k-\omega S S T$ model and its buoyancy-modified version reveals that a better agreement with experimental data is obtained for the original model. In case of a buoyancy-modified $k-\omega$ SST model, larger values for $\bar{u}$ near the bottom are observed after wave breaking $(x \geq 7.275 \mathrm{~m})$. Based on the RMSE values, the buoyancy-modified $k-\omega$ model gives the best comparison with the experimental measurements, in particular for $x \geq 7.275 \mathrm{~m}$.

A common issue when applying RANS turbulence modelling is the overprediction of TKE inside the flow domain (see section 1). This is confirmed by the profiles presenting $\bar{k}$ for the original $k-\omega$ SST model 
(see Figure 3). For each profile, large deviations are visually observed and a significant RMSE is calculated between numerical and experimental data compared to the buoyancy-modified models. For the first three sampling locations however, no experimental data is available because no turbulence was expected [27]. This is confirmed by the results using the buoyancy-modified $k-\omega$ SST model showing very small values of $\bar{k}$ along the water column. Remarkably, the buoyancy-modified $k-\omega$ model returns only small values for $\bar{k}$ at the first sampling location ( $x=-1.265 \mathrm{~m}$ ). If the buoyancy term is implemented in the TKE-equation, the numerically predicted $\bar{k}$ approaches the experimental determined values for $x \geq 7.275 \mathrm{~m}$. In particular for the buoyancy-modified $k$ - $\omega$ SST model, excellent predictions are obtained similar to the experimental measurements. Strangely, the better predictions for $\bar{k}$ do not result in better profiles for $\bar{u}$, on the contrary.

\subsubsection{Turbulent behaviour}

In order to identify the behaviour of the different RANS turbulence models around the breaking point, contour plots of the turbulent kinematic viscosity $v_{t}$ at different time phases are depicted in Figure 4 using a logarithmic scale. In case no buoyancy term is implemented in the TKE-equation, the turbulent kinematic viscosity in every computational cell is several orders of magnitudes larger than the kinematic viscosity of water $\left(10^{-6} \mathrm{~m}^{2} / \mathrm{s}\right)$. However, if the density is explicitly included in the turbulence transport equations and a buoyancy term is added to the TKE-equation, two observations are made. Firstly, prior to wave breaking, i.e. wave propagation, the turbulent kinematic viscosity around the free water surface goes to zero. Secondly, around the breaking point, the turbulent kinematic viscosity around the free water surface is several orders of magnitudes larger than the kinematic viscosity of water. This means that there is a strong turbulent flow field at the free water surface where wave breaking occurs. A comparison between the buoyancy-modified $k-\omega$ and $k-\omega$ SST model shows some significant differences. For example, $v_{t}$ in the water column is predicted larger for the buoyancymodified $k-\omega$ model compared to the buoyancy-modified $k-\omega S S T$ model. This is again explained by the limiter inside the production term of TKE for the $k-\omega$ SST model.

Furthermore, Figure 4 depicts contour plots of the magnitude of the Reynolds stress tensor (equation (9)) using a linear scale for cells with a volume fraction $\alpha$ between 0.5 and 1 (i.e. cells below the free water surface). The magnitude of the Reynolds stress tensor is a good indicator to show where turbulence is present in the flow field. In general, a different spatial variation of the magnitude of the Reynolds stress tensor in the flow field is predicted by the three turbulence models. However for each model, turbulence is generated in the crest when the waves are breaking. The largest magnitudes are observed for the original $k$ - $\omega$ SST model while the buoyancy-modified $k$ - $\omega$ model predicts slightly larger magnitudes compared to the buoyancy-modified $k-\omega$ SST model. Moreover, turbulence is only observed in the upper part of the water column and spreads out slowly downwards. Interestingly, a large amount of entrapped air just below the free water surface is only observed for the buoyancymodified $k$ - $\omega$ SST model. Clearly, the smaller values of $\bar{k}$ have their effect on the more vivid (less damping) breaking process simulated involving bursts, splashes and more entrapped air.

\subsection{Plunging breakers}

In this subsection, tests are performed in case of plunging breakers. Similar graphs are reported as shown in section 3.1 for the case of spilling breakers. Again, surface elevations along the wave flume, time averaged horizontal velocities and TKE along several vertical profiles and the turbulent behaviour of the flow using different RANS turbulence models are presented.

\subsubsection{Surface elevations}

Surface elevations along the wave flume are depicted in Figure 5 for the different numerical simulations performed. Remarkably, at first sight, the solution without turbulence model provides the 
best fit with the experimental data: the breaking point is correctly predicted and the breaking wave height is overestimated at the same level as the turbulent solutions. However, this solution should be avoided for two reasons. Firstly, there is a large standard deviation along the complete length of the wave flume, putting a burden on the wave-by-wave repeatability of the result. Secondly, as was the case for the spilling breakers, the average and minimum surface elevations are clearly underpredicted. In general, better results are obtained if a RANS turbulence model is applied. For example, the standard deviation decreases significantly over the length of the flume, except for the buoyancy-modified $k-\omega$ SST model after the waves broke. However, the breaking wave height is still overpredicted and also the numerically obtained breaking point is before the experimental observation. Similar to spilling breakers, deviations between numerical and experimental results are only observed for the maximum surface elevations and not for the average and minimum ones. These observations are not valid for the original $k-\omega$ model which shows again wave damping over the length of the flume. In contrast to the observations made for spilling breakers, the $k-\omega$ model including only the density is not showing significant wave damping. Probably, the lower wave steepness of the plunging breakers in the part with constant water depth $(H / L=0.0128)$ is not triggering wave damping as observed for the spilling breakers (steepness $H / L=0.0388$ ) (cfr. wave propagation simulations for non-breaking waves in [33]). In general, the buoyancy-modified $k-\omega$ model has again the best performance for the surface elevations in case of plunging breakers despite its slightly larger RMSE value for the maximum surface elevations $E_{\max }$ compared to the other models.

\subsubsection{Undertow profiles}

Figure 6 shows both the measured and simulated vertical profiles (i.e. time averaged horizontal velocity $\bar{u}$ and time averaged turbulent kinetic energy (TKE) $\bar{k}$ ) at locations $x=7.295 \mathrm{~m}, x=7.795 \mathrm{~m}$, $x=8.345 \mathrm{~m}, x=8.795 \mathrm{~m}, x=9.295 \mathrm{~m}, x=9.795 \mathrm{~m}$ and $x=10.395 \mathrm{~m}$ (see vertical dashed lines in Figure $5)$. Again, the numerical results using no turbulence model and the original $k-\omega$ model are excluded because the predicted surface elevations included in Figure $5 \mathrm{a}$ and Figure $5 \mathrm{~b}$ are inaccurate.

Along the different profiles, the sign of $\bar{u}$ is predicted correctly by all three turbulent simulations. Again, the largest deviations between experimental and numerical results are found in the vicinity of the bottom, especially for $x \geq 8.345 \mathrm{~m}$. In particular for the buoyancy-modified $k-\omega$ SST model, significantly overestimated values for $\bar{u}$ are calculated. This is also observed for the spilling breakers reported in section 3.1.2. As a result, the performance of the buoyancy-modified $k-\omega$ model for $\bar{u}$ is substantially better compared to the other turbulence models based on the RMSE values.

Also for plunging breakers, an overprediction of TKE inside the flow domain is observed in case the original $k$ - $\omega S S T$ model is applied. This is revealed by the overpredicted values of $\bar{k}$ along the different vertical profiles. The RMSE values decrease significantly in case a buoyancy-modified turbulence model is used. In particular, the performance of the buoyancy-modified $k-\omega S S T$ model is better than the buoyancy-modified $k$ - $\omega$ model regarding the values of $\bar{k}$ along the different profiles. Interestingly, this better behaviour is more pronounced at the first four sampling locations shown in Figure 6 (see $x \leq$ $8.795 \mathrm{~m})$. Again, better predictions for $\bar{k}$ are not reflected in the velocity field.

\subsubsection{Turbulent behaviour}

The behaviour of the different RANS turbulence models under plunging breakers is examined by Figure 7 in which contour plots of the turbulent kinematic viscosity $v_{t}$ are visualised using a logarithmic scale at different time phases around the breaking point. Similar observations are made as reported in section 3.1.3 for the spilling breakers. A buoyancy term is needed in order to force the turbulent kinematic viscosity around the free water surface to zero in case of wave propagation. If the wave breaks, the turbulent kinematic viscosity around the free water surface is several orders of magnitudes 
larger than the kinematic viscosity of water $\left(10^{-6} \mathrm{~m}^{2} / \mathrm{s}\right)$, indicating a strong turbulent flow field. Moreover, it is again observed that $v_{t}$ in the water column is predicted larger for the buoyancymodified $k-\omega$ model compared to the buoyancy-modified $k-\omega S S T$ model.

Subsequently, the Reynolds stress tensor is calculated by equation (9) and its magnitude is visualised in Figure 7 by contour plots using a linear scale for cells with a volume fraction $\alpha$ between 0.5 and 1 . Similar to spilling breakers, the spatial variation of the magnitude of the Reynolds stress tensor in the flow field differs between the three turbulence models. Each buoyancy-modified turbulence model indicates that turbulence is generated by the impact of the overturning volume of water on the free water surface resulting in splash. This is also observed for the original $k-\omega S S T$ model, but turbulence is already present before wave breaking. Again, the original $k-\omega S S T$ model predicts the largest magnitudes followed by the buoyancy-modified $k-\omega$ model and the buoyancy-modified $k-\omega S S T$ model. More importantly, turbulence is observed in a large part of the water column, even near the bottom. In case of plunging breakers, significantly more air is entrapped and for the buoyancy-modified $k-\omega S S T$ model even observed near the bottom of the numerical wave flume.

\section{Discussion}

In general, the numerical results are in a very good agreement with the experimental data and with similar numerical studies for both spilling and plunging breakers, not only for the surface elevations along the wave flume but also for the undertow profiles. Most importantly, enhanced predictions are obtained for the TKE in the flow field compared to other numerical studies. Furthermore, the behaviour of the flow field in the present numerical study is correctly predicted in the way reported by Ting and Kirby [2]:

- The turbulence levels are much higher in case of plunging breakers compared to spilling breakers;

- The variations of $\bar{u}$ and $\bar{k}$ in the vertical profiles are smaller for plunging breakers compared to spilling breakers;

- TKE in the surf zone decreases towards the bottom.

The most important observation lies in the influence of the buoyancy modification. The results show indeed that the improved turbulence models behave as expected. Observations of the turbulent kinematic viscosity are in line with the initial assumptions of the performance of the buoyancy term implemented in the TKE-equation:

- The buoyancy term $G_{b}$ avoids wave damping in the wave propagation zone by inducing a laminar solution of the flow field near the free water surface (see [33]);

- The buoyancy term $G_{b}$ goes to zero in the wave breaking zone and the original turbulence model (including the density) is regained near the free water surface;

- Furthermore, the buoyancy term $G_{b}$ is not the direct cause of better predictions for TKE. However, it is observed that better predictions are obtained, in particular for TKE, with the buoyancy-modified turbulence models. In fact, the buoyancy term will only limit the build-up of TKE around the free water surface in the wave propagation zone (since a laminar solution is induced around the free water surface). This results in a lower and thus better prediction of TKE in the surf zone.

- Moreover, the influence of $G_{b}$ on the results of TKE is much smaller for the $k-\omega S S T$ model than for the $k-\omega$ model due to the limiter in the production term $P_{k}$ of TKE for the $k-\omega$ SST model. That limiter is in general reducing the build-up of TKE independent of the inclusion of the buoyancy term. 
Despite the improvements, the solution is still not perfect and small discrepancies are observed between the numerically obtained surface elevations and the experimental measurements. Firstly as mentioned in section 1, a lot of measurement difficulties exist in small-scale experimental tests. Secondly, numerical models involves discretisation and interpolation errors. Thirdly, post processing of the numerically obtained data also contributes to the interpolation error (e.g. evaluation of the surface elevations). For both spilling and plunging breakers, larger differences of the maximum surface elevations along the wave flume have been noticed compared to the average and minimum ones. This may be related to air entrainment in the upper parts of the water column caused by the wave breaking process. It is also observed that more air is entrapped, even down to the bottom region, for the plunging breakers (see Figure 4 and Figure 7). The amount of entrapped air has a direct influence on the determination of the surface elevations due to a discrete integration of the volume fraction. Possibly, this explains why larger differences for the maximum surface elevations are observed compared to the experimental data for the plunging breakers. Moreover, a number of discrepancies between experimental and numerical data are observed for the undertow profiles for $\bar{u}$ and vertical profiles for $\bar{k}$. This is possibly correlated to a slightly different breaking point shifting the correspondent profile towards a slightly different location. Furthermore, it is expected that the inaccuracies related to the experimental measurements also contribute to the achieved level of agreement.

Lastly, the turbulence models themselves are not designed for the highly transient two-phase flow during wave breaking. Redesigning the turbulence models would require more validation data (TKE, but also Reynolds stresses, dissipation rate, etc.), which is scarce. Nevertheless, the discrepancies found are in line with other reported numerical results and we explicitly refer to the recent paper by Brown et al. [27]. In general, the results of our study using the buoyancy-modified turbulence models and the results using the same models reported by Brown et al. are fairly similar. More specifically regarding the surface elevations, the results are almost identical. For example, the breaking point is similar for the two numerical studies. Recall that Brown et al. also included the density in the turbulence transport equations, but did not introduce the buoyancy term. We notice that by comparing our results using the buoyancy term with Brown's results without (figures 5, 6, 8 and 9 in [27]), a major improvement is found for the time averaged horizontal velocities $\bar{u}$ and time averaged turbulent kinetic energy $\bar{k}$. An accurate prediction of the latter is necessary to correctly calculate the turbulent viscosity. In particular, the turbulent viscosity will be of extreme importance once a movable bed is considered involving sediment transport under the action of wave induced bed shear stresses.

\section{Conclusions}

In this paper, we evaluated the performance of both a buoyancy-modified $k-\omega$ and a $k-\omega$ SST model for simulating breaking waves using OpenFOAM. The obtained numerical results of the surface elevations, undertow profiles and TKE levels show a good agreement with the experimental data for both spilling and plunging breakers. Moreover, the benefits of modifying existing turbulence models for buoyancy effects have been revealed. Firstly, in the flow field prior to wave breaking (i.e. during wave propagation), low turbulence levels are observed and a laminar solution is desirable. We demonstrated that the buoyancy term forces the solution of the flow field near the free water surface to a laminar solution in case of wave propagation. This also avoids wave damping over the length of the flume due to RANS turbulence modelling. Secondly in the surf zone where waves break, significant turbulence levels are noticed. For this zone, the buoyancy term goes to zero and a fully turbulent flow field is resolved by the numerical model. For all the simulations presented, we conclude that the results predicted by the buoyancy-modified turbulence models agree the best with the experimental measurements. In particular, the buoyancy-modified turbulence models significantly reduce the common overestimation of TKE in the flow field. Furthermore, we also conclude that the best 
performance for simulating breaking waves is obtained with the buoyancy-modified $k-\omega$ model compared to the other models tested in this paper. Moreover, we demonstrated that the inclusion of the buoyancy term is essential for a $k-\omega$ model.

The outcome of this study jointly with [33], proves the successful capability of buoyancy-modified turbulence models to simulate offshore and coastal engineering processes. The buoyancy-modified turbulence models not only result in a stable wave propagation model without wave damping but also their predicted turbulence levels inside the flow field are in a better agreement with the experimental measurements in the surf zone.

\section{Acknowledgement}

The first author is Ph.D. fellow of the Research Foundation - Flanders (FWO), Belgium (Ph.D. fellowship $1133817 \mathrm{~N})$.

\section{References}

[1] C.J. Galvin, Breaker type classification on three laboratory beaches, J. Geophys. Res. 73 (1968) 3651-3659. doi:10.1029/JB073i012p03651.

[2] F.C.K. Ting, J.T. Kirby, Observation of undertow and turbulence in a laboratory surf zone, Coast. Eng. 24 (1994) 51-80. doi:10.1016/0378-3839(94)90026-4.

[3] M. Boers, Simulation of a Surf Zone with a Barred Beach; Part 1: Wave Heights and Wave Breaking, 1996.

[4] D.T. Cox, N. Kobayashi, Identification of intense, intermittent coherent motions under shoaling and breaking waves, J. Geophys. Res. Ocean. 105 (2000) 14223-14236. doi:10.1029/2000JC900048.

[5] F.C.K. Ting, Laboratory study of wave and turbulence velocities in a broad-banded irregular wave surf zone, Coast. Eng. 43 (2001) 183-208. doi:10.1016/S0378-3839(01)00013-8.

[6] F.C.K. Ting, Laboratory study of wave and turbulence characteristics in narrow-band irregular breaking waves, Coast. Eng. 46 (2002) 291-313. doi:10.1016/S0378-3839(02)00092-3.

[7] F.C.K. Ting, Large-scale turbulence under a solitary wave, Coast. Eng. 53 (2006) 441-462. doi:10.1016/j.coastaleng.2005.11.004.

[8] F.C.K. Ting, Large-scale turbulence under a solitary wave: Part 2, Coast. Eng. 55 (2008) 522-536. doi:10.1016/j.coastaleng.2008.02.018.

[9] Z.-C. Huang, H.-H. Hwung, S.-C. Hsiao, K.-A. Chang, Laboratory observation of boundary layer flow under spilling breakers in surf zone using particle image velocimetry, Coast. Eng. 57 (2010) 343-357. doi:10.1016/j.coastaleng.2009.11.004.

[10] F.C.K. Ting, J.R. Nelson, Laboratory measurements of large-scale near-bed turbulent flow structures under spilling regular waves, Coast. Eng. 58 (2011) 151-172. doi:10.1016/j.coastaleng.2010.09.004.

[11] B.M. Sumer, M.B. Sen, I. Karagali, B. Ceren, J. Fredsøe, M. Sottile, L. Zilioli, D.R. Fuhrman, Flow and sediment transport induced by a plunging solitary wave, J. Geophys. Res. 116 (2011) C01008. doi:10.1029/2010JC006435.

[12] F.C.K. Ting, Laboratory measurements of large-scale near-bed turbulent flow structures under plunging regular waves, Coast. Eng. 77 (2013) 120-139. doi:10.1016/j.coastaleng.2013.02.014. 
[13] D.A. van der A, J. van der Zanden, T. O’Donoghue, D. Hurther, I. Cáceres, S.J. McLelland, J.S. Ribberink, Large-scale laboratory study of breaking wave hydrodynamics over a fixed bar, J. Geophys. Res. Ocean. 122 (2017) 3287-3310. doi:10.1002/2016JC012072.

[14] N.G. Jacobsen, J. Fredsoe, J.H. Jensen, Formation and development of a breaker bar under regular waves. Part 1: Model description and hydrodynamics, Coast. Eng. 88 (2014) 182-193. doi:10.1016/j.coastaleng.2013.12.008.

[15] N.G. Jacobsen, J. Fredsoe, Formation and development of a breaker bar under regular waves. Part 2: Sediment transport and morphology, Coast. Eng. 88 (2014) 55-68. doi:10.1016/j.coastaleng.2014.01.015.

[16] A. Fernandez-Mora, J.S. Ribberink, J. van der Zanden, J.J. van der Werf, N.G. Jacobsen, RANSVOF modeling of hydrodynamics and sand transport under full-scale non-breaking and breaking waves, Coast. Eng. Proc. 1 (2017) 29. doi:10.9753/ICCE.V35.SEDIMENT.29.

[17] Z. Zhou, T.-J. Hsu, D. Cox, X. Liu, Large-eddy simulation of wave-breaking induced turbulent coherent structures and suspended sediment transport on a barred beach, J. Geophys. Res. Ocean. 122 (2017) 207-235. doi:10.1002/2016JC011884.

[18] P. Lin, P.L.F. Liu, A numerical study of breaking waves in the surf zone, J. Fluid Mech. 359 (1998) 239-264. doi:10.1017/S002211209700846X.

[19] S.F. Bradford, Numerical Simulation of Surf Zone Dynamics, J. Waterw. Port, Coastal, Ocean Eng. 126 (2000) 1-13. doi:10.1061/(ASCE)0733-950X(2000)126:1(1).

[20] S. Mayer, P.A. Madsen, Simulation of Breaking Waves in the Surf Zone using a Navier-Stokes Solver, Coast. Eng. (2000) 928-941. doi:http://dx.doi.org/10.1061/40549(276)72.

[21] E.D. Christensen, Large eddy simulation of spilling and plunging breakers, Coast. Eng. 53 (2006) 463-485. doi:10.1016/j.coastaleng.2005.11.001.

[22] P.D. Hieu, T. Katsutoshi, V.T. Ca, Numerical simulation of breaking waves using a two-phase flow model, Appl. Math. Model. 28 (2004) 983-1005. doi:10.1016/j.apm.2004.03.003.

[23] N.G. Jacobsen, D.R. Fuhrman, J. Freds $\varnothing$ e, A wave generation toolbox for the open-source CFD library: OpenFoam ${ }^{\circledR}$, Int. J. Numer. Methods Fluids. 70 (2012) 1073-1088. doi:10.1002/fld.2726.

[24] Z. Xie, Two-phase flow modelling of spilling and plunging breaking waves, Appl. Math. Model. 37 (2013) 3698-3713. doi:10.1016/j.apm.2012.07.057.

[25] M. Alagan Chella, H. Bihs, D. Myrhaug, M. Muskulus, Breaking characteristics and geometric properties of spilling breakers over slopes, Coast. Eng. 95 (2015) 4-19. doi:10.1016/j.coastaleng.2014.09.003.

[26] M. Alagan Chella, H. Bihs, D. Myrhaug, M. Muskulus, Hydrodynamic characteristics and geometric properties of plunging and spilling breakers over impermeable slopes, Ocean Model. 103 (2016) 53-72. doi:10.1016/j.ocemod.2015.11.011.

[27] S.A. Brown, D.M. Greaves, V. Magar, D.C. Conley, Evaluation of turbulence closure models under spilling and plunging breakers in the surf zone, Coast. Eng. 114 (2016) 177-193. doi:10.1016/j.coastaleng.2016.04.002.

[28] E.B. Thornton, Energetics of breaking waves within the surf zone, J. Geophys. Res. 84 (1979) 4931. doi:10.1029/JC084iC08p04931.

[29] S.B. Pope, Turbulent flows, Cambridge University Press, 2000. 
[30] F.R. Menter, Zonal Two Equation k- $\omega$ Turbulence Models For Aerodynamic Flows, in: 23rd Fluid Dyn. Plasmadynamics, Lasers Conf., American Institute of Aeronautics and Astronautics, Reston, Virigina, 1993. doi:10.2514/6.1993-2906.

[31] D.C. Wilcox, Turbulence modeling for CFD, DCW Industries, Inc., La Canada, California, 1998.

[32] F.R. Menter, Influence of freestream values on k- $\omega$ turbulence model predictions, AIAA J. 30 (1992) 1657-1659. doi:10.2514/3.11115.

[33] B. Devolder, P. Rauwoens, P. Troch, Application of a buoyancy-modified k- $\omega$ SST turbulence model to simulate wave run-up around a monopile subjected to regular waves using OpenFOAM $^{\circledR}$, Coast. Eng. 125 (2017) 81-94. doi:10.1016/j.coastaleng.2017.04.004.

[34] K. Van Maele, B. Merci, Application of two buoyancy-modified k- $\varepsilon$ turbulence models to different types of buoyant plumes, Fire Saf. J. 41 (2006) 122-138. doi:10.1016/j.firesaf.2005.11.003.

[35] K. Van Maele, B. Merci, Importance of buoyancy and chemistry modelling in steady RANS simulations of well-ventilated tunnel fires, Turkish J. Eng. Environ. Sci. 30 (2006) 145-155.

[36] OpenFOAM ${ }^{\circledR}$, OpenFOAM-2.2.2, (2013).

[37] E. Berberović, N.P. van Hinsberg, S. Jakirlić, I. V. Roisman, C. Tropea, Drop impact onto a liquid layer of finite thickness: Dynamics of the cavity evolution, Phys. Rev. E. 79 (2009) 36306. doi:10.1103/PhysRevE.79.036306.

[38] C.W. Hirt, B.D. Nichols, Volume of Fluid (VoF) Method for the Dynamics of Free Boundaries, J. Comput. Phys. 39 (1981) 201-225. doi:10.1016/0021-9991(81)90145-5.

[39] F.R. Menter, J.C. Ferreira, T. Esch, The SST Turbulence Model with Improved Wall Treatment for Heat Transfer Predictions in Gas Turbines, Int. Gas Turbine Congr. 2003. (2003) 1-7.

[40] V. Vukčević, H. Jasak, I. Gatin, Implementation of the Ghost Fluid Method for free surface flows in polyhedral Finite Volume framework, Comput. Fluids. 153 (2017) 1-19. doi:10.1016/j.compfluid.2017.05.003.

[41] D.B. Spalding, A Single Formula for the "Law of the Wall," J. Appl. Mech. 28 (1961) 455. doi:10.1115/1.3641728.

[42] P. Higuera, J.L. Lara, I.J. Losada, Realistic wave generation and active wave absorption for Navier-Stokes models. Application to OpenFOAM., Coast. Eng. 71 (2013) 102-118. doi:10.1016/j.coastaleng.2012.07.002.

[43] P. Higuera, J.L. Lara, I.J. Losada, Simulating coastal engineering processes with OpenFOAM., Coast. Eng. 71 (2013) 119-134. doi:10.1016/j.coastaleng.2012.06.002.

[44] B. van Leer, Towards the ultimate conservative difference scheme. II. Monotonicity and conservation combined in a second-order scheme, J. Comput. Phys. 14 (1974) 361-370. doi:10.1016/0021-9991(74)90019-9.

[45] N.G. Jacobsen, A Full Hydro- and Morphodynamic Description of Breaker Bar Development, Technical University of Denmark, 2011. 


\section{Figures}

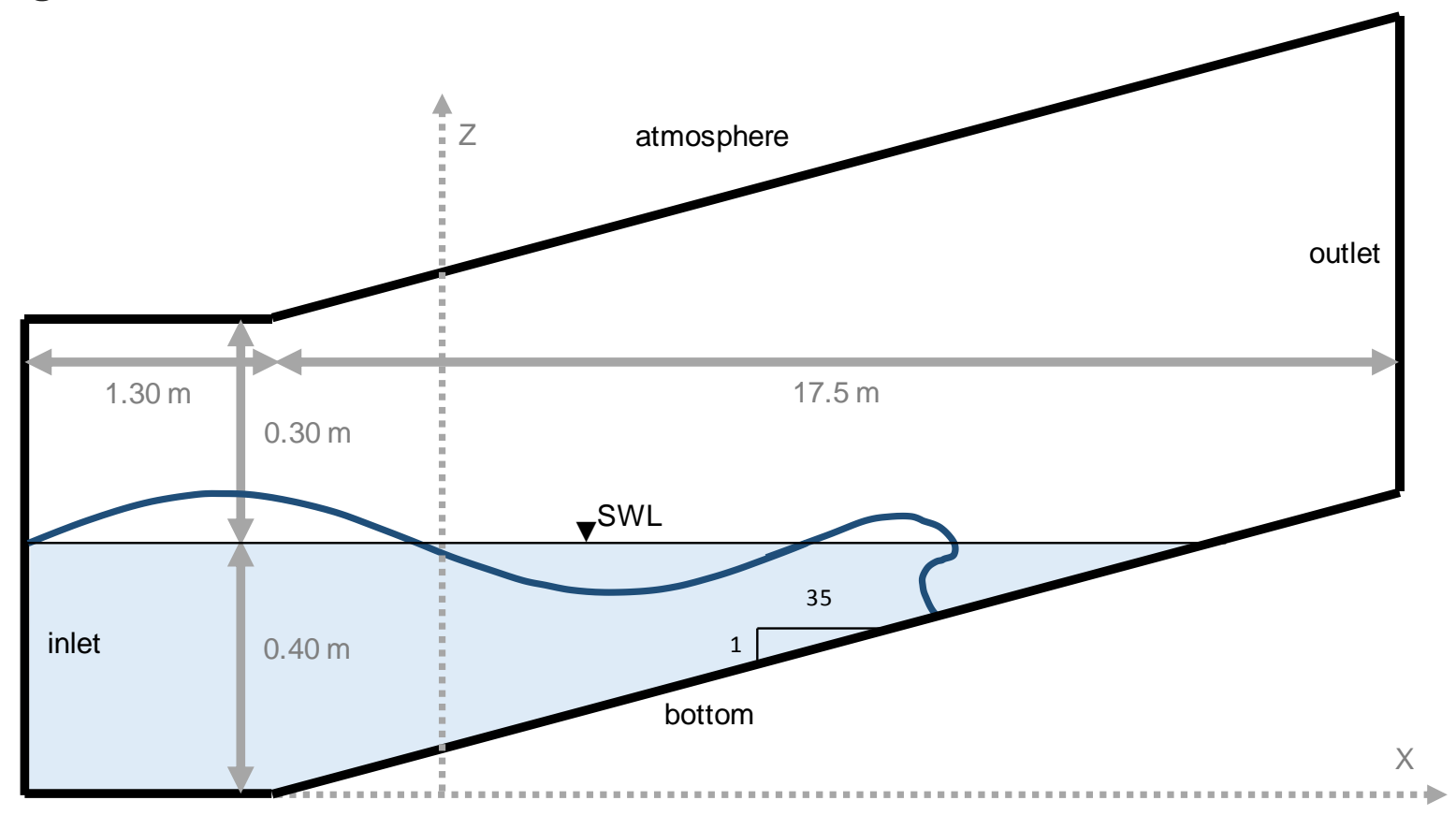

Figure 1: Definition sketch of the 2DV computational domain (in the XZ-plane). The thin horizontal line indicates the still water level (SWL), with water depth $d=0.40 \mathrm{~m}$ at the inlet, whereas the black words characterise the boundary condition type. The grey arrows denote the key distances (at distorted scale). 


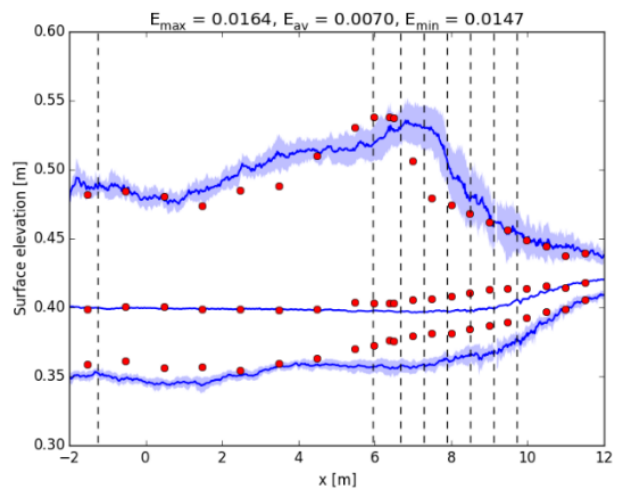

(a) no turbulence model

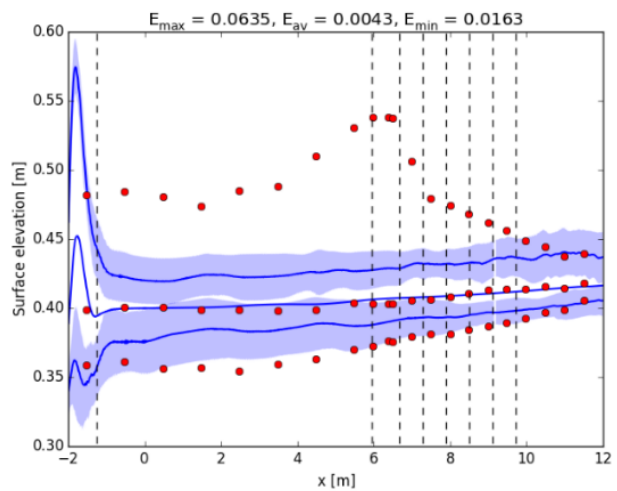

(b) original $k-\omega$

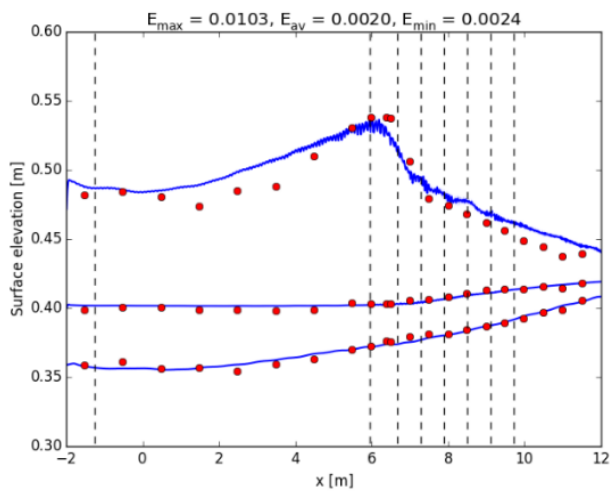

(e) original $k-\omega S S T$

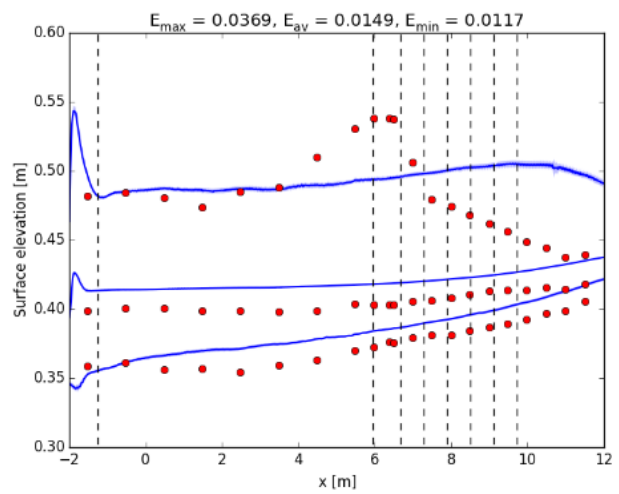

(c) $k$ - $\omega$ including only the density

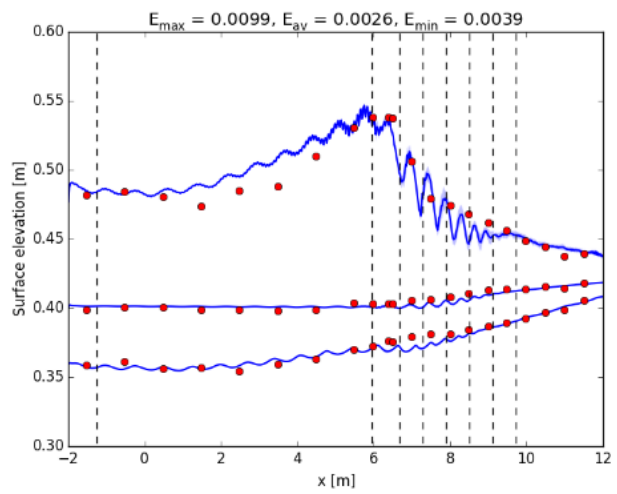

(f) $k-\omega S S T$ including only the density

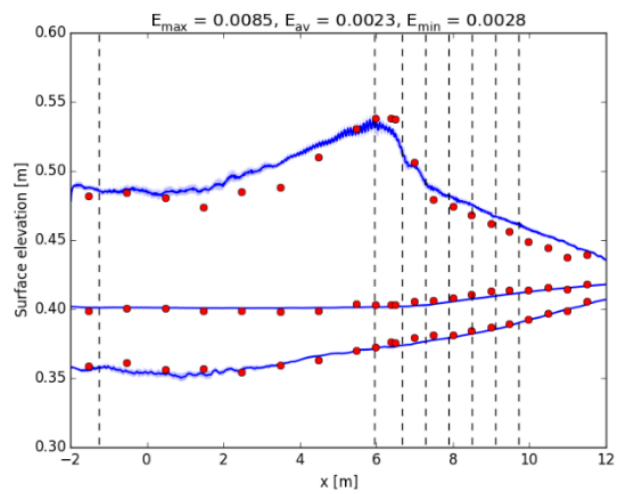

(d) buoyancy-modified $k-\omega$

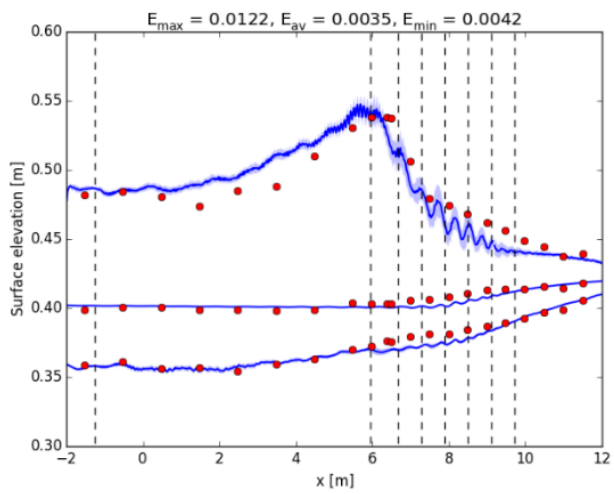

(g) buoyancy-modified $k-\omega S S T$

Figure 2: Numerically obtained surface elevations averaged over 20 wave periods along the wave flume for the case of spilling breakers using (a) no turbulence model (b) the original $k-\omega$ (c) $k-\omega$ including only the density (d) the buoyancy-modified $k$ - $\omega$ (e) the original $k-\omega S S T$ (f) $k$ - $\omega$ SST including only the density $(g)$ the buoyancy-modified $k$ - $\omega$ SST model. The solid blue lines depict the maximum, average and minimum phase averaged surface elevations. On both sides of the maximum and minimum surface elevations, one standard deviation is visualised by a blue shaded band. RMSE values, $E[\mathrm{~m}]$, are calculated for the maximum, average and minimum surface elevations with respect to the experimental data [2], represented by the red dots. The vertical dashed black lines indicate the position where the undertow profiles are extracted, see section 3.1.2. 


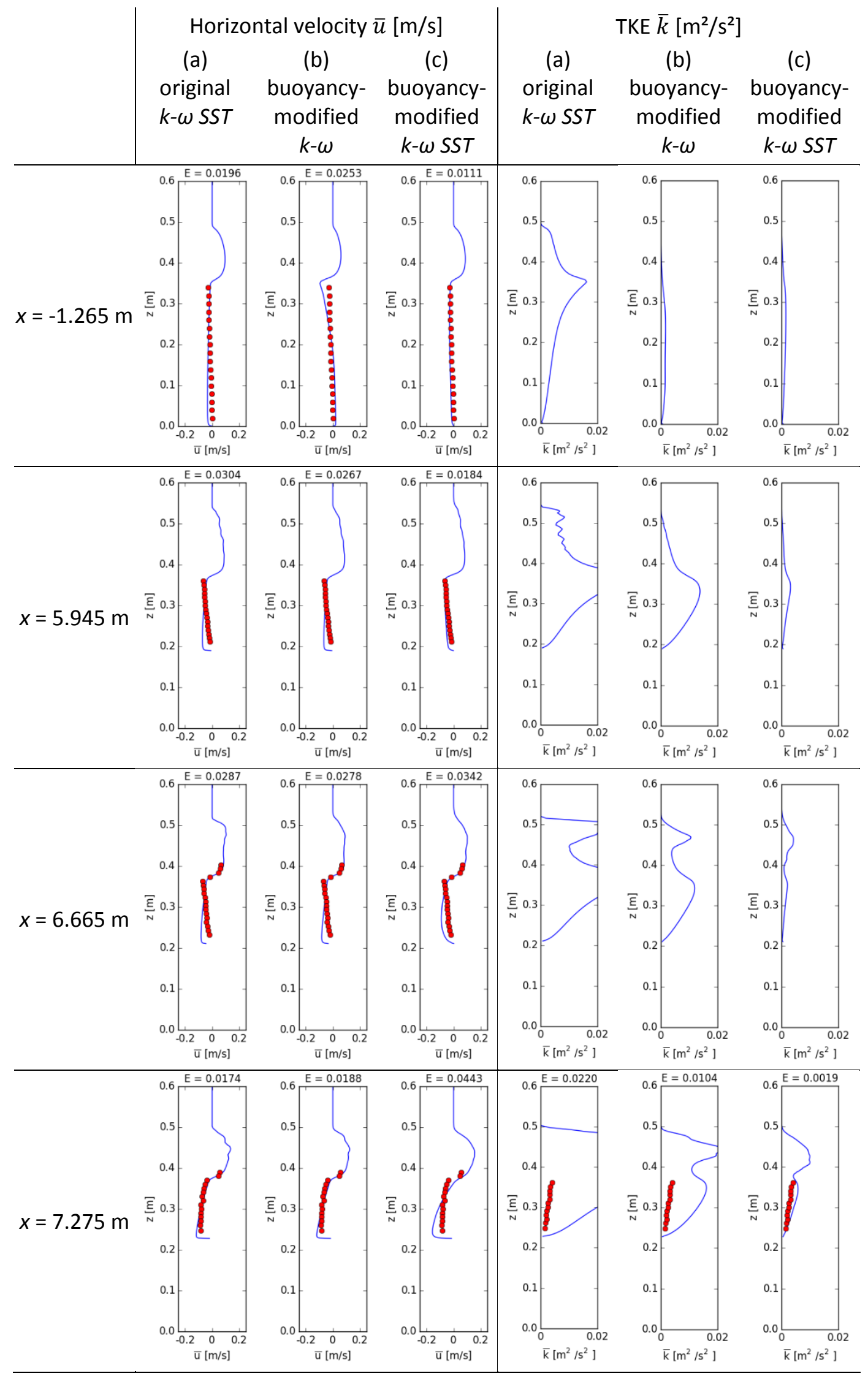




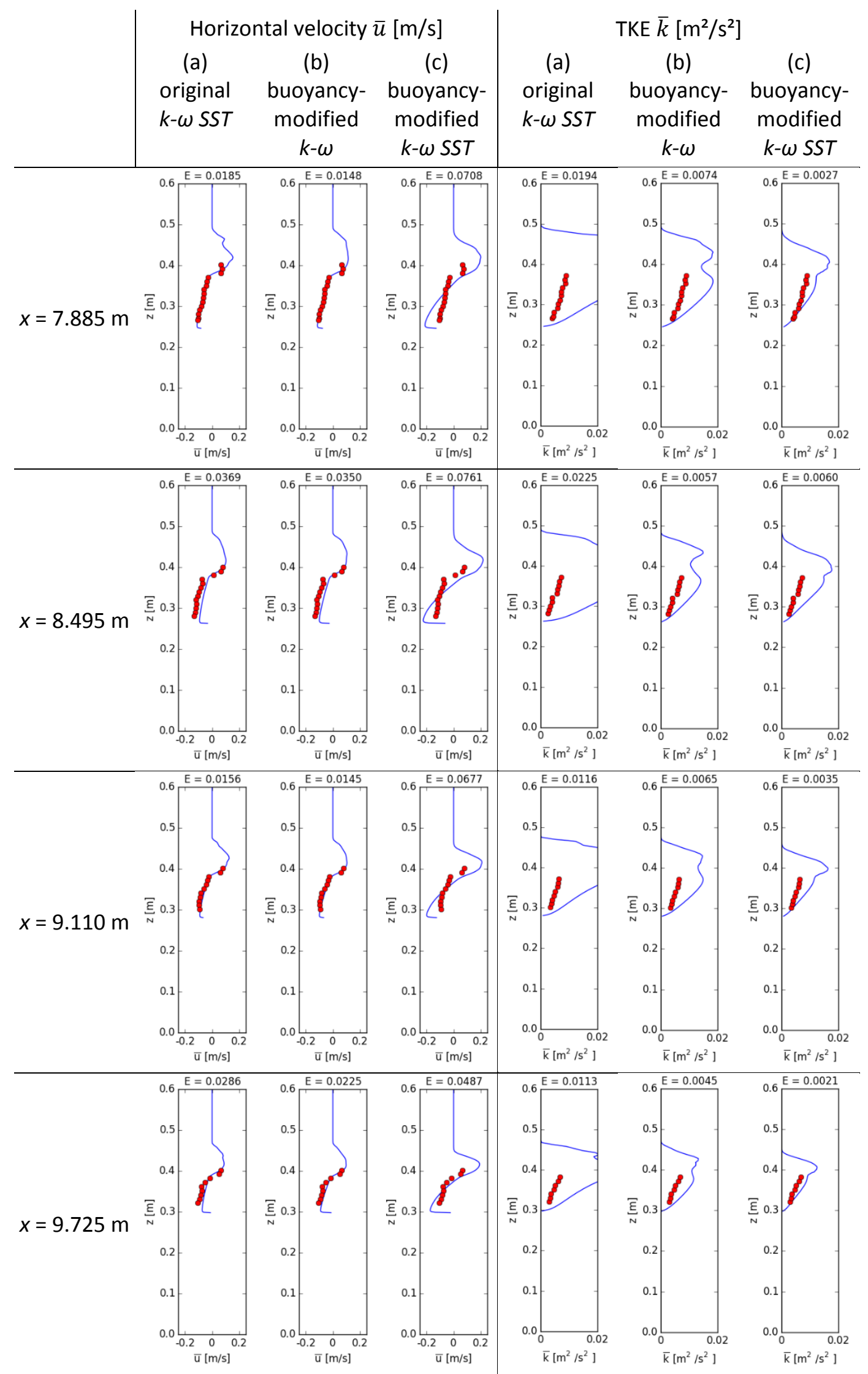

Figure 3: Numerically obtained undertows for the time averaged horizontal velocity $\bar{u}$ and time averaged TKE $\bar{k}$ (blue lines) averaged over 20 wave periods using the (a) buoyancy-modified $k-\omega(b)$ original $k$ - $\omega$ SST (c) buoyancy-modified k- $\omega$ SST model. The red dots represent the experimental data [2] for the case of spilling breakers. On top of each curve, RMSE values are denoted by $E\left([\mathrm{~m} / \mathrm{s}]\right.$ for $\bar{u}$ and $\left[\mathrm{m}^{2} / \mathrm{s}^{2}\right]$ for $\left.\bar{k}\right)$. 
Turbulent kinematic viscosity
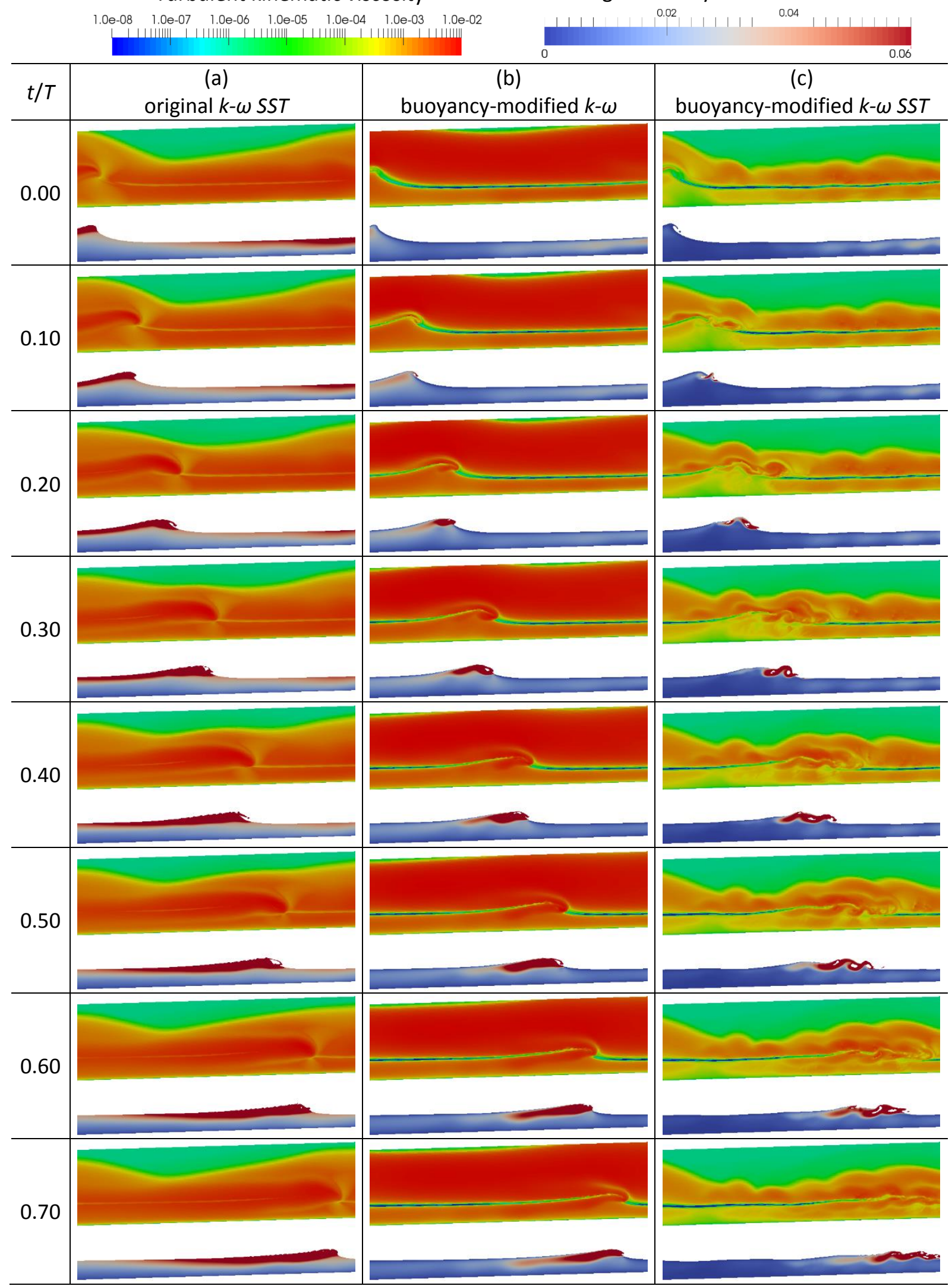

Figure 4: Numerically obtained snapshots around the breaking point of the turbulent kinematic viscosity $v_{t}\left[\mathrm{~m}^{2} / \mathrm{s}\right]$ and the magnitude of the Reynolds stress tensor (equation (9)) at different time phases for the case of spilling breakers using the (a) original k- $\omega$ SST (b) buoyancy-modified k- $\omega$ (c) buoyancy-modified k- $\omega$ SST model.

Magnitude Reynolds stress tensor

$0.02+0.04$ 


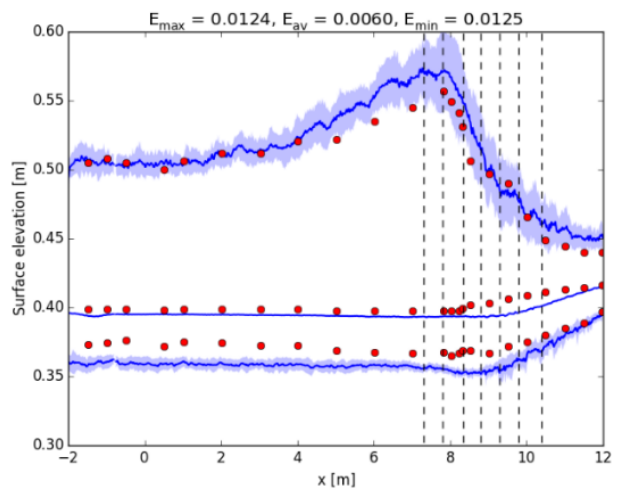

(a) no turbulence model

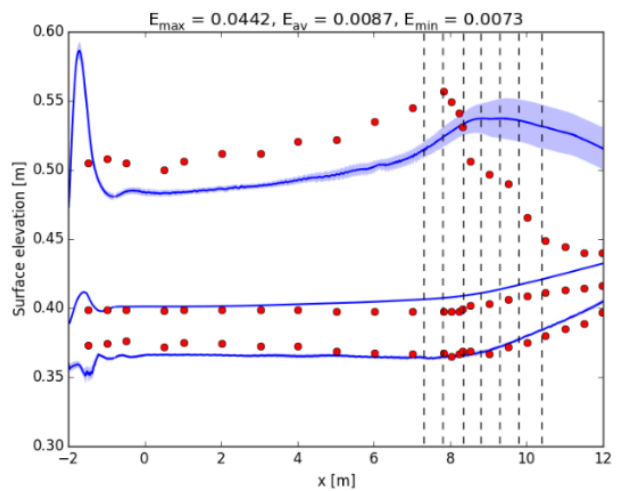

(b) original $k-\omega$

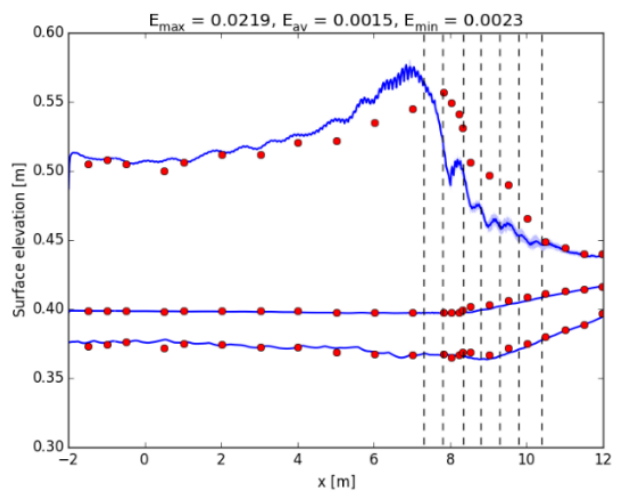

(e) original $k-\omega S S T$

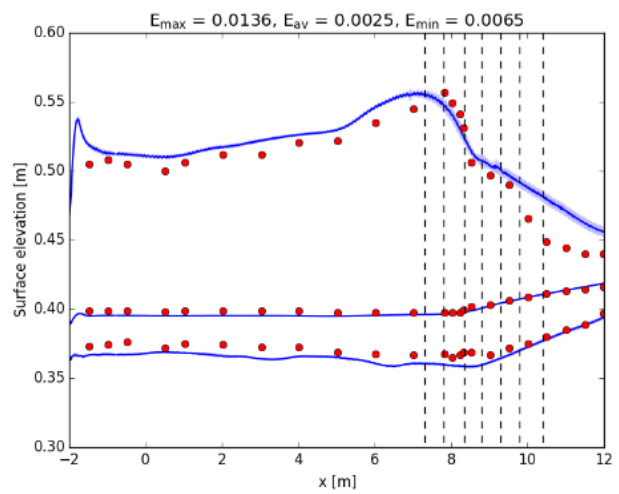

(c) $k$ - $\omega$ including only the density

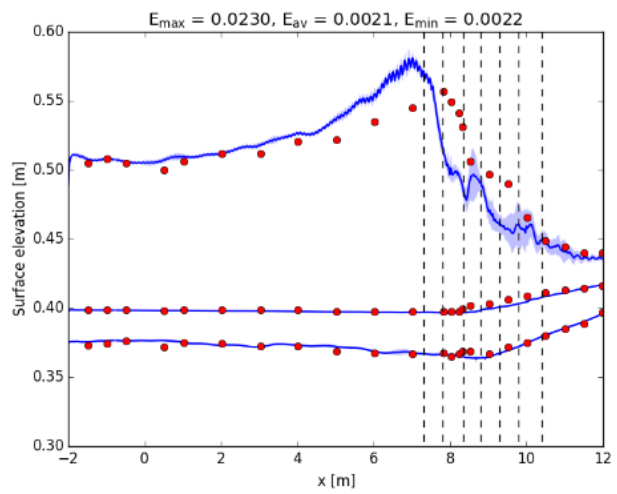

(f) $k-\omega S S T$ including only the density

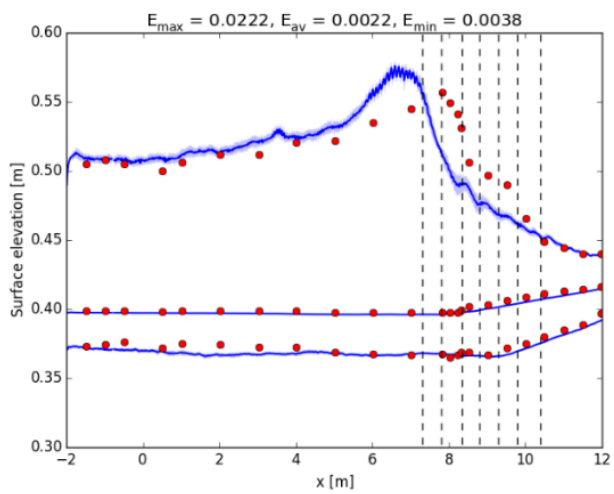

(d) buoyancy-modified $k-\omega$

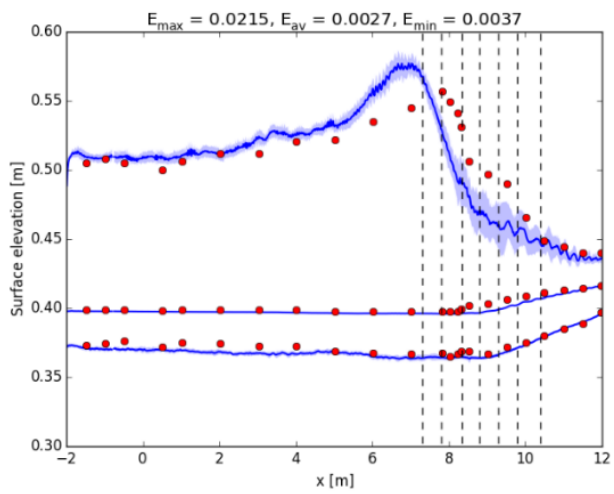

(g) buoyancy-modified $k-\omega S S T$

Figure 5: Numerically obtained surface elevations averaged over 20 wave periods along the wave flume for the case of plunging breakers using (a) no turbulence model (b) the original $k-\omega$ (c) $k$ - $\omega$ including only the density (d) the buoyancymodified $k-\omega(e)$ the original $k-\omega S S T$ ( $f$ ) $k$ - $\omega$ SST including only the density $(g)$ the buoyancy-modified $k-\omega S S T$ model. The solid blue lines depict the maximum, average and minimum phase averaged surface elevations. On both sides of the maximum and minimum surface elevations, one standard deviation is visualised by a blue shaded band. RMSE values, $E$ [m], are calculated for the maximum, average and minimum surface elevations with respect to the experimental data [2], represented by the red dots. The vertical dashed black lines indicate the position where the undertow profiles are extracted, see section 3.2.2. 


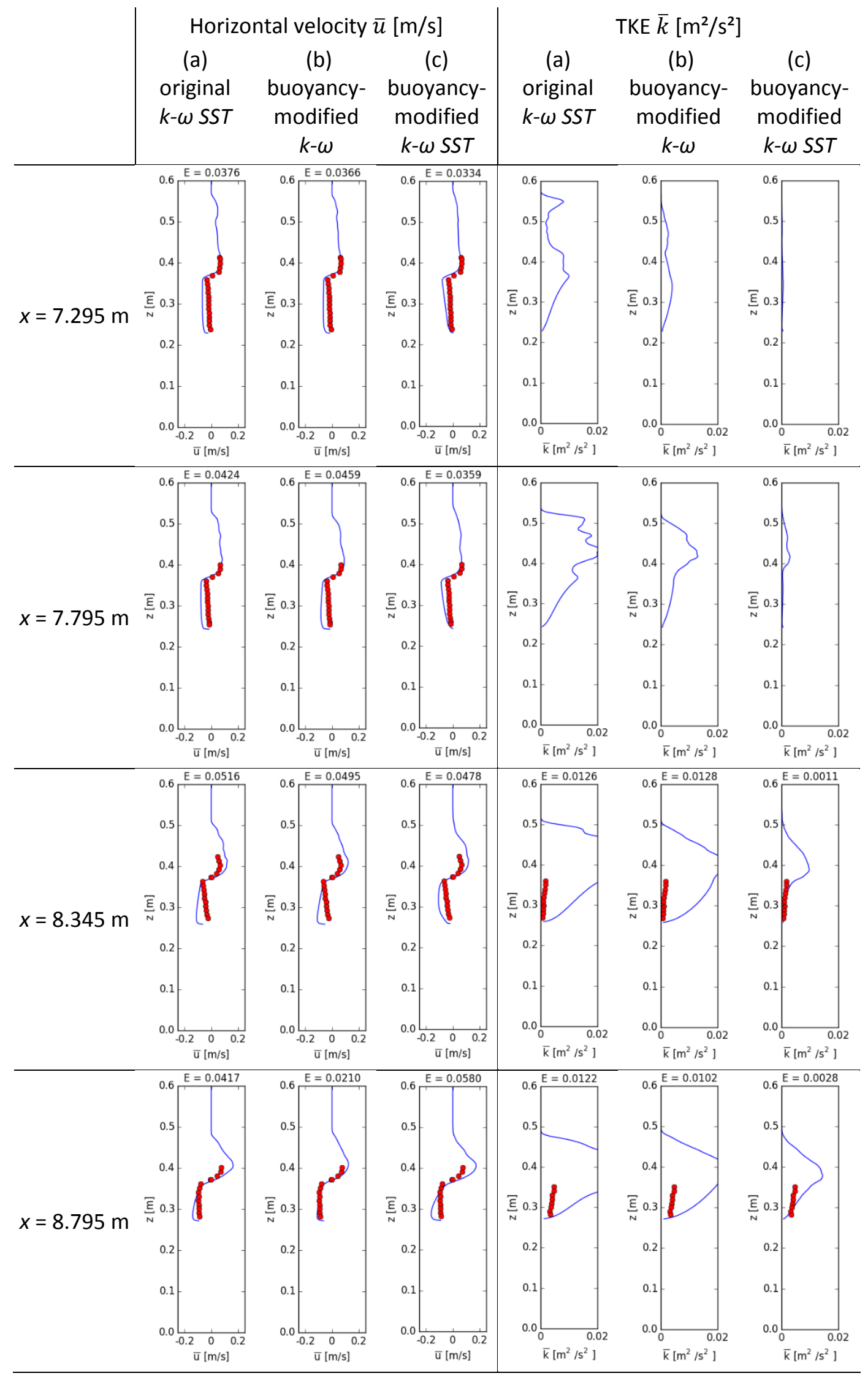




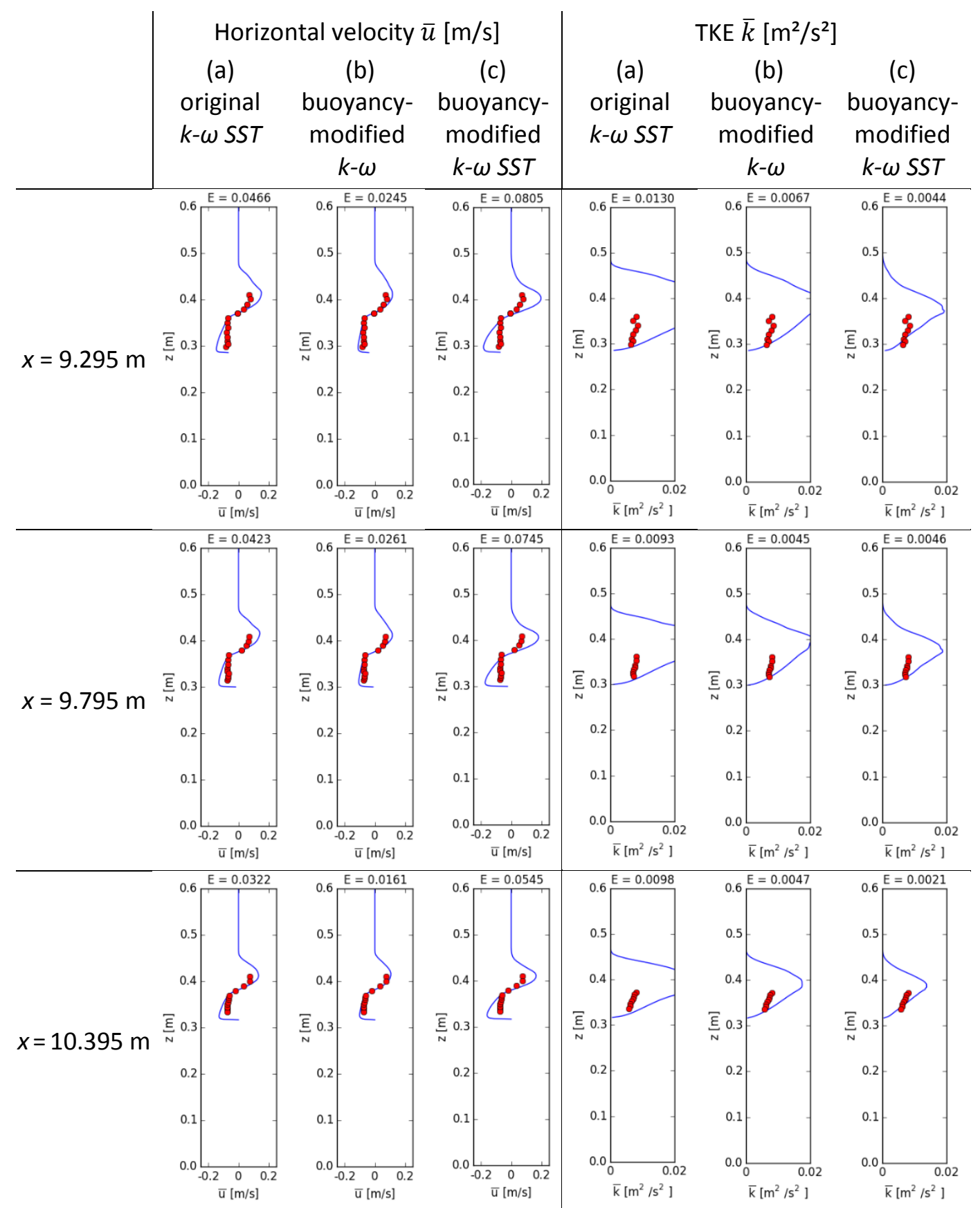

Figure 6: Numerically obtained undertows for the time averaged horizontal velocity $\bar{u}$ and time averaged TKE $\bar{k}$ (blue lines) averaged over 20 wave periods using the (a) buoyancy-modified $k-\omega$ (b) original $k-\omega$ SST (c) buoyancy-modified $k$ - $\omega$ SST model. The red dots represent the experimental data [2] for the case of plunging breakers. On top of each curve, RMSE values are denoted by $E\left([\mathrm{~m} / \mathrm{s}]\right.$ for $\bar{u}$ and $\left[\mathrm{m}^{2} / \mathrm{s}^{2}\right]$ for $\left.\bar{k}\right)$. 
Turbulent kinematic viscosity

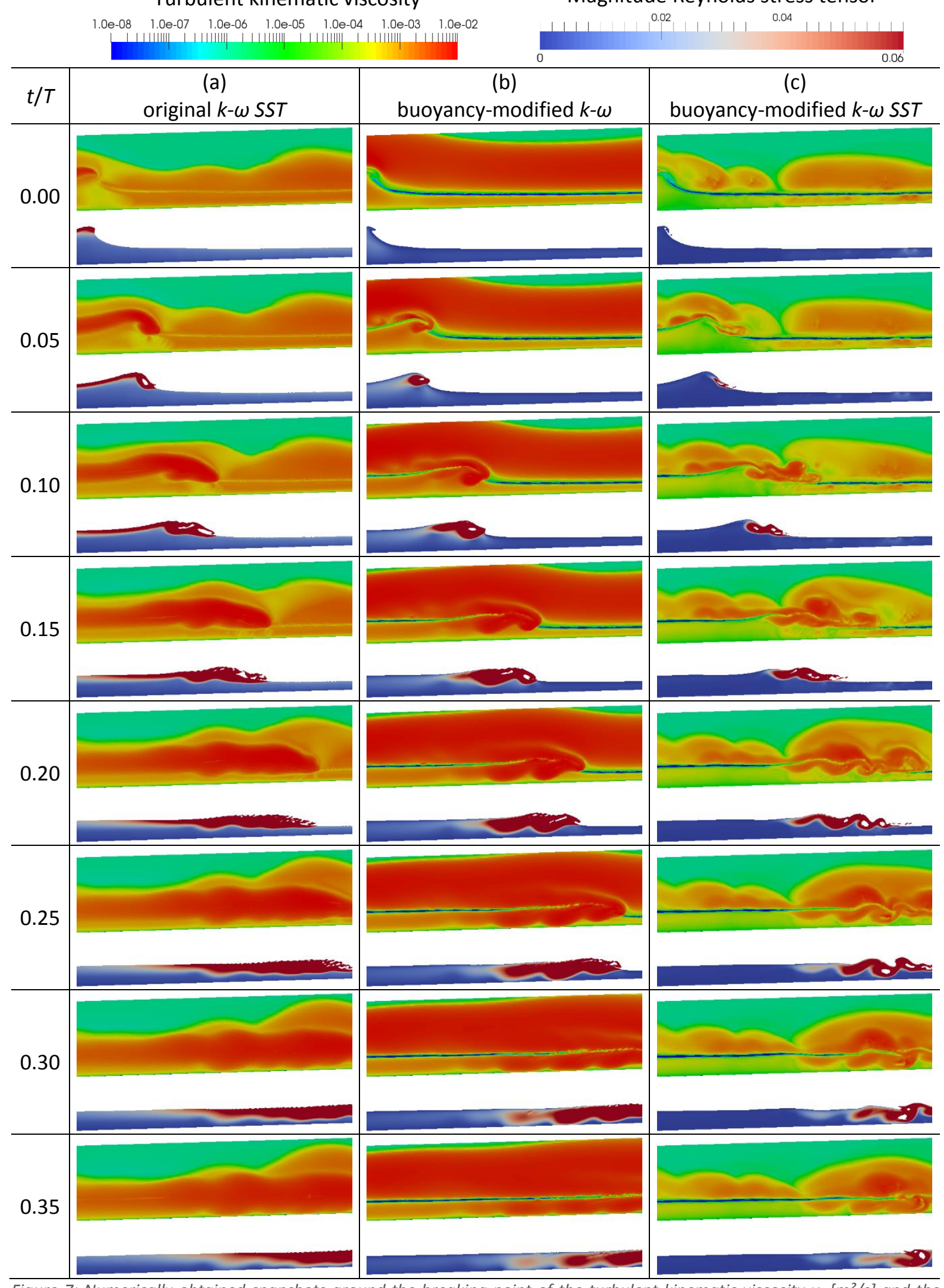

Figure 7: Numerically obtained snapshots around the breaking point of the turbulent kinematic viscosity $v_{+}\left[\mathrm{m}^{2} / \mathrm{s}\right]$ and the magnitude of the Reynolds stress tensor (equation (9)) at different time phases for the case of plunging breakers using the (a) original $k-\omega$ SST (b) buoyancy-modified $k-\omega(c)$ buoyancy-modified $k-\omega$ SST model. 